\title{
Evaluation of Zinc Oxide Nano-Microtetrapods for Biomolecule Sensing Applications
}

WEI ZHAO 



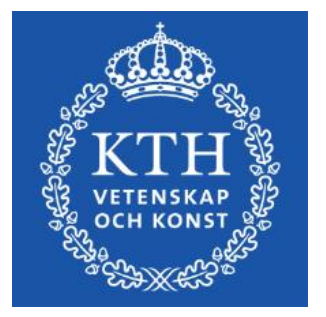

\title{
SWEDISH \\ ש \\ ACREO
}

\section{Evaluation of Zinc Oxide Nano- Microtetrapods for Biomolecule Sensing Applications}

\author{
Wei Zhao \\ Submitted in partial fulfillment of the requirements for the degree of \\ Master of Science in Nanotechnology
}

Division of Functional Materials (FNM)

School of Information and Communication Technology (ICT)

KTH Royal Institute of Technology

Stockholm, Sweden, 2015 
Postal Address

KTH Royal Institute of Technology

Division of Functional Materials (FNM)

Electrum 229, Isafjordsgatan 22

SE 16 440, Stockholm, Sweden

Examiner

Prof. Muhammet S. Toprak

KTH Royal Institute of Technology

toprak@kth.se

Supervisor

Dr. Qin Wang

Acreo Swedish ICT AB

gin.wang@acreo.se

Co-supervisor

Yichen Zhao

KTH Royal Institute of Technology

yichen@kth.se

TRITA-ICT-EX-2015:233 
Dedicated to my parents 



\section{ABSTRACT}

Zinc oxide $(\mathrm{ZnO})$ is a well-known II-VI semiconductor material that has gained renewed interest in the past decade due to the developments of growth technologies and the availability of high-quality $\mathrm{ZnO}$ bulk single crystals. Owing to a wide direct band gap (3.37 $\mathrm{eV})$, large exciton binding energy $(60 \mathrm{meV})$, and high electron mobility $\left(440 \mathrm{~cm}^{2} \mathrm{~V}^{-1} \mathrm{~s}^{-1}\right)$, $\mathrm{ZnO}$ has been used for applications including actuators, optoelectronics, and sensors. $\mathrm{ZnO}$ nanoparticles can be synthesized in a broad variety of morphologies, such as nanotetrapods, nanotubes, and nanowires. Among these nanostructures, the tetrapods have attracted significant attention due to their unique morphology consisting of four legs connected together in a tetrahedral symmetry. Recently, it has been reported that nano-microstructured $\mathrm{ZnO}$ tetrapods (ZnO-Ts) can be synthesized by flame transport synthesis (FTS) in a rapid and up-scalable approach. Compared to conventional $\mathrm{ZnO}$ nanoparticles, the nanomicrostructured $\mathrm{ZnO}-\mathrm{Ts}$ can reduce cellular uptake, while still exhibiting specific nanomaterial properties due to the nanoscale tips. Moreover, the anisotropic $\mathrm{ZnO}$-Ts have the advantages of multiple electron transfer paths, chemical stability, and biocompatibility, which make the $\mathrm{ZnO}$-Ts promising candidates for biomolecule sensing applications.

This work herein reports a systematical study on the structural, optical and electrochemical properties of the ZnO-Ts, which were synthesized by FTS using precursor $\mathrm{Zn}$ microparticles. The morphology of the ZnO-Ts was confirmed by scanning electron microscopy (SEM) as joint structures of four single crystalline legs, of which the diameter of each leg is $0.7-2.2 \mu \mathrm{m}$ in average from the tip to the stem. The $\mathrm{ZnO}-\mathrm{Ts}$ were dispersed in glucose solutions to study the photoluminescence as well as photocatalytic activity in a mimicked biological environment. The photoluminescence (PL) intensity in the ultraviolet (UV) region decreased with linear dependence on the glucose concentration up to $4 \mathrm{mM}$. The $\mathrm{ZnO}$-Ts were also attached with glucose oxidase $(\mathrm{GOx})$ and over coated with Nafion ${ }^{\circledR}$ to form the active media for electrochemical glucose sensing. The active layers were confirmed by Fourier transform infrared spectroscopy (FT-IR). Furthermore, the current response of the active layers to glucose was studied by cyclic voltammetry (CV) in various glucose concentration conditions. Stable current response to glucose was detected with linear dependence on the glucose concentration up to $12 \mathrm{mM}$, which confirms the potential of $\mathrm{ZnO}-\mathrm{Ts}$ for biomolecule sensing applications.

Keywords: zinc oxide tetrapods, nano-microstructure, glucose oxidase, glucose sensor 


\section{ACKNOWLEDGEMENTS}

I gladly express my sincere gratitude to Professor Muhammet S. Toprak for his valuable guidance, support and motivation. Professor Toprak was the inspiration that fueled my interest to continue my studies in the field of nanotechnology. I feel privileged to have been a part of the Division of Functional Materials (FNM) at KTH. I am grateful for the warm welcome that I have received, and the opportunity to work in the chemistry lab independently and to learn many characterization tools. My time in the group is just the beginning of my academic journey and I am convinced we will meet again in the future.

I would also express my sincere gratitude to Dr. Qin Wang for providing me the opportunity to perform my thesis work at Acreo Swedish ICT AB. Dr. Wang has played a central role in shaping and leading the direction of this work. Apart from her valuable guidance, love, and dedication, she is also an expert on baking delicious desserts that we all appreciated during our seminar meetings. Moreover, she has introduced me to two international conferences and supported me all the way. We shared joyful memories in Riga, Latvia at the EuroNanoForum2015 conference on nanotechnology. I wish we can share more joyful memories in the future.

Likewise, I express my sincere gratitude to PhD student Yichen Zhao, who was always there for me during this period. She has been a strong link and made sure that we maintained a continuous work flow and communication between all involved members. The time and energy she has contributed is beyond words. She has taught me a wide range of skills from lab routines and operation of characterization tools to scientific writing and well-designed PPT-presentations. Without her contribution and supervision, this work would not have been possible. In the time of her $\mathrm{PhD}$ thesis writing, I wish her the best and hope we will meet again in the future.

In parallel to this work, my colleague Mikael Karlsson also worked on his Master's thesis with a similar project topic; graphene-based glucose sensor. Together we have supported and learned from each other. I sincerely appreciate the time with Mikael during this period, all the challenges we have overcome in the lab with twisted ideas, discussions about the universe, and coffee breaks after midnight outside the lab. Moreover, it was 
rewarding to attend the SPIE Micro+Nano Materials, Devices, and Applications 2015 in Sydney, Australia; an international conference where we both delivered a session talk. There could not have been a better companion alongside during this period.

My time in FNM has also allowed me to meet new friends, who have shown their love, care and support. In hard times, they have made my days colorful. I am grateful to have met Bejan, Srejith, Adem, Yuxuan, Chonmanart, Rabia, Natalia, Ahmet, Xiaoyu, Maoxiang, Homayoun, Amin, Tanjim, Joydip, Shafiullah, Katayoun, and Arash.

Above all, my final words and my deepest gratitude are expressed to my parents. Their love, encouragement and support have given me the strength to complete this work during hard times. It is with love and respect I dedicate this work to my parents. 


\section{LIST OF FIGURES}

Figure 1. Number of publications based on $\mathrm{ZnO}$ from 1950 to present. SOURCE: Web of Science. 3

Figure 2. Hexagonal $\mathrm{ZnO}$ wurtzite structure from side view and top view, with the elements $\mathrm{Zn}$ (purple) and $\mathrm{O}$ (red). The 3D structures were drawn in 3D Crystal Viewer.

Figure 3. GOx with two FAD (yellow) and next to it is a single FAD in fully oxidized form, with elements $\mathrm{C}$ (grey), $\mathrm{O}$ (red), $\mathrm{N}$ (blue), and $\mathrm{P}$ (pink). The 3D structures were drawn in ChemBio3D Ultra. .7

Figure 4. The evolution of the first to the third generation of electrochemical glucose biosensors.

Figure 5. 3D model of a) gold-coated wafer and b) six lesser $0.5 \mathrm{~cm}$ x $1.0 \mathrm{~cm}$ gold-coated substrates.

Figure 6. Schematic illustration of the pull-off test configuration and the procedure. .....12

Figure 7. Preparation steps for 3D layer-by-layer Au/ZnO-Ts/GOx/Nafion electrode for electrochemical glucose sensing.

Figure 8. SEM micrographs of a) ZnO-Ts networks and b) ZnO-Ts interconnections. ... 15

Figure 9. SEM micrograph of a) 3D interconnected networks of $\mathrm{ZnO}-\mathrm{Ts}$ and b) corresponding size distribution summarized from the diameter of the tips and stems. (Inset: Single $\mathrm{ZnO}-\mathrm{T}$ ).

Figure 10. TEM micrograph of a) single ZnO-T tip and b) SAED pattern of that tip. 16 
Figure 11. PL emission spectra of a) ZnO-Ts in DI water without and with $5 \mathrm{mM}$ glucose, and b) schematic diagram of the proposed PL emission and intensity quenching mechanism.

Figure 12. PL emission spectra of a) $\mathrm{ZnO}-\mathrm{Ts}$ in $\mathrm{DI}$ water with various glucose concentrations and b) PL intensity change of the NBE versus glucose concentration relative to that of the $\mathrm{ZnO}-\mathrm{Ts}$ without glucose.

Figure 13. PL emission spectra of a) $\mathrm{ZnO}-\mathrm{Ts}$ in $\mathrm{DI}$ water with various hydrogen peroxide concentrations and b) PL intensity change of the NBE versus hydrogen peroxide concentration relative to that of the $\mathrm{ZnO}-\mathrm{Ts}$ without hydrogen peroxide

Figure 14. Photographs of a) $\mathrm{ZnO}-\mathrm{Ts}$ suspension and b) 3D layer-by-layer $\mathrm{Au} / \mathrm{ZnO}-\mathrm{Ts}$ electrode.

Figure 15. Photographs of a) GOx solution and b) 3D layer-by-layer Au/ZnOTs/GOx/Nafion electrode.

Figure 16. SEM micrograph of a) Au/ZnO-Ts/GOx electrode and b) FT-IR spectra of $\mathrm{Au} / \mathrm{ZnO}-\mathrm{Ts}$ electrode without and with GOx. (Inset: Single $\mathrm{ZnO}-\mathrm{T}$ coated with GOx) 22

Figure 17. SEM micrograph of a) 3D layer-by-layer Au/ZnO-Ts/GOx/Nafion electrode and b) FT-IR reflectance spectra of $\mathrm{Au} / \mathrm{ZnO}-\mathrm{Ts} / \mathrm{Nafion}$ electrode without and with GOx. (Inset: Single ZnO-T coated with GOx and Nafion ${ }^{\circledR}$ ). 22

Figure 18. Schematic diagram of the electrochemical cell, potentiostat, and PC for inputs and monitoring.

Figure 19. Photograph of a) three-electrode configuration comprising bare gold WE, $\mathrm{Ag} / \mathrm{AgCl} \mathrm{RE}$ and platinum $\mathrm{CE}$, and b) cyclic voltammogram of the bare gold electrode in PBS from $-1.0 \mathrm{~V}$ to $1.0 \mathrm{~V}$ at the scan rate of $50 \mathrm{mV} / \mathrm{s}$. 
Figure 20. Cyclic voltammogram of a) Au/Nafion electrode in PBS without and with ZnOTs at the scan rate of $50 \mathrm{mV} / \mathrm{s}$, and b) $\mathrm{Au} / \mathrm{ZnO}-\mathrm{Ts} / \mathrm{GO} / \mathrm{Nafion}$ electrode in PBS without and with $5 \mathrm{mM}$ glucose.

Figure 21. Cyclic voltammogram of a) Au/ZnO-Ts/GOx/Nafion electrode in PBS with 5 $\mathrm{mM}$ glucose measured at scan rate from 50-100 $\mathrm{mV} / \mathrm{s}$, and b) the anodic and cathodic peak current versus the square root of the scan rate. 25

Figure 22. Cyclic voltammogram of a) Au/ZnO-Ts/GOx/Nafion electrode and the current response to various glucose concentrations at the scan rate of $50 \mathrm{mV} / \mathrm{s}$, and d) the deduced calibration curve of current response versus glucose concentration from $1 \mathrm{mM}$ to $40 \mathrm{mM}$ at fixed potential $0.6 \mathrm{~V}$. .26 


\section{LIST OF TABLES}

Table 1. Pull-off test of gold-coated substrate without and with annealing treatment. ...20 


\section{ABBREVIATIONS}

OD

1D

2D

3D

CE

$\mathrm{CV}$

DI

DLE

FAD

FS

FT-IR

GOx

$\mathrm{H}_{2} \mathrm{O}_{2}$

NBE

PBS

PL

RE

SEM

SM

TEM

UV

UV-vis

WE

$\mathrm{ZnO}$

ZnO-Ts
Zero-dimensional

One-dimensional

Two-dimensional

Three-dimensional

Counter electrode

Cyclic voltammetry

Deionized

Deep-level emission

Flavin adenine dinucleotide

Fluorescence spectrometer

Fourier transform infrared spectroscope

Glucose oxidase

Hydrogen peroxide

Near-band edge emission

Phosphate buffered saline

Photoluminescence

Reference electrode

Scanning tunneling microscope

Stereo microscope

Tunneling electron microscope

Ultravioet

Ultraviolet-visible spectrometer

Working electrode

Zinc oxide

Zinc oxide tetrapods 


\section{CONTENTS}

ABSTRACT

ACKNOWLEDGEMENTS

1. Introduction

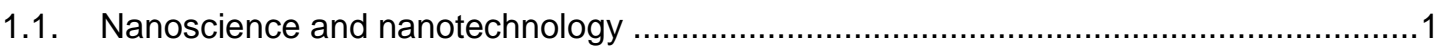

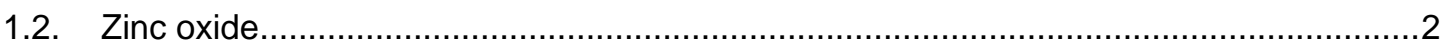

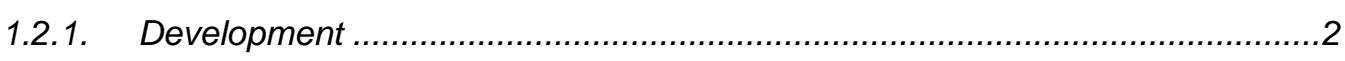

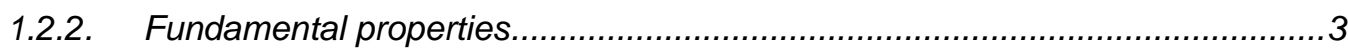

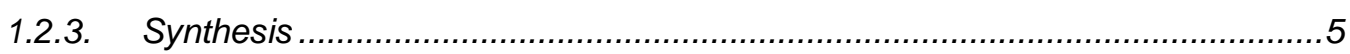

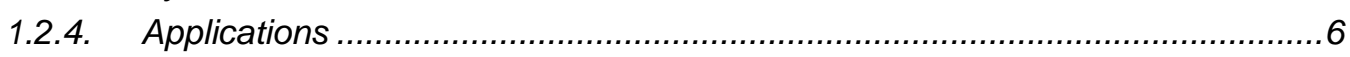

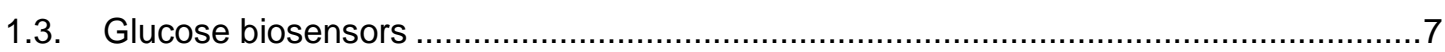

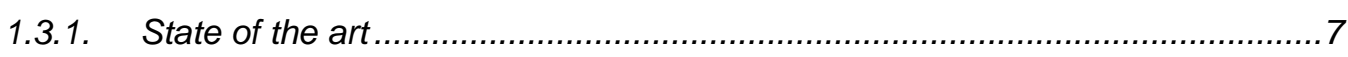

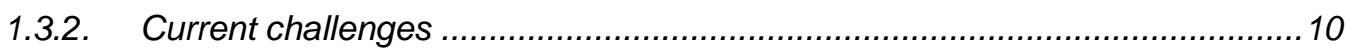

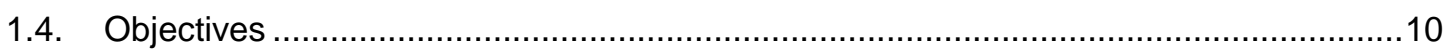

2. Experimental details $\quad 11$

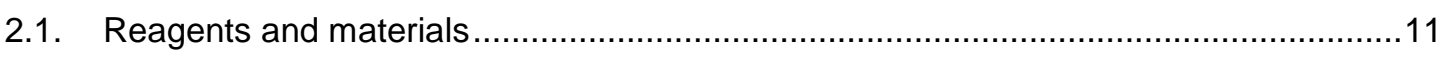

2.2. ZnO tetrapods for electrochemical glucose sensing ..................................................11

2.2.1. Fabrication of gold-coated substrates ....................................................11

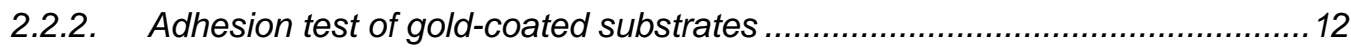

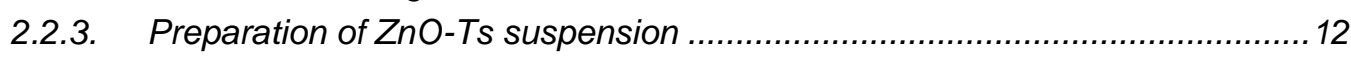

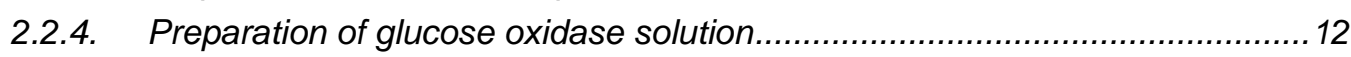

2.2.5. Assembly of the Au/ZnO-Ts/GOx/Nafion electrode .....................................13

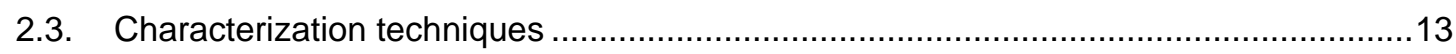

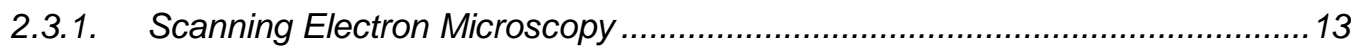

2.3.2. Transmission Electron Microscopy ............................................................13

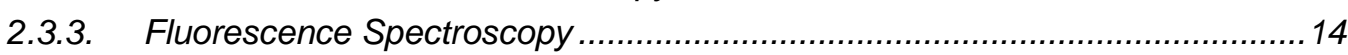

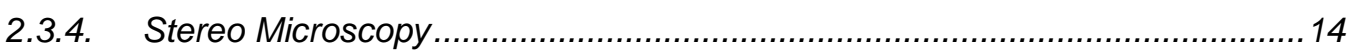

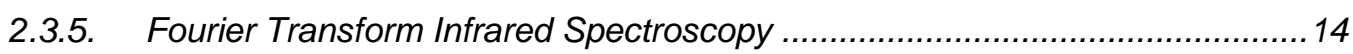

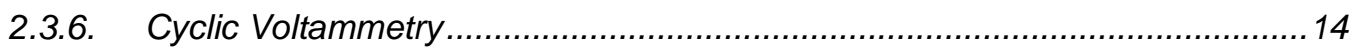


3. Results and discussion 15

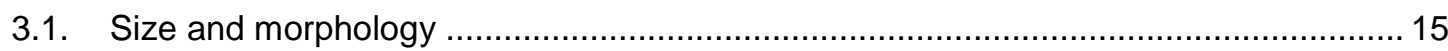

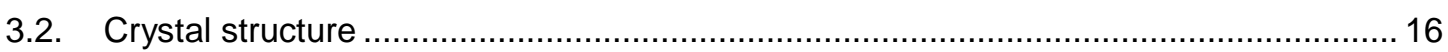

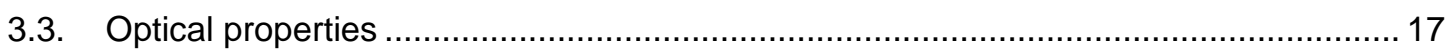

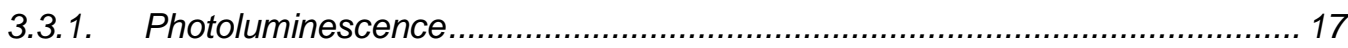

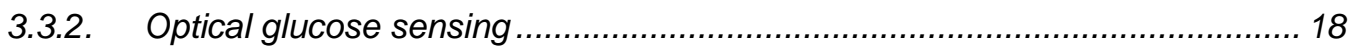

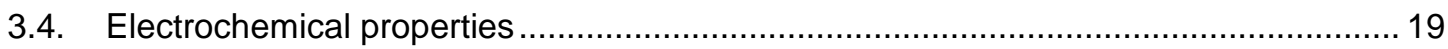

3.4.1. Adhesion test of gold-coated substrates................................................... 19

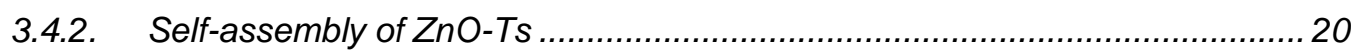

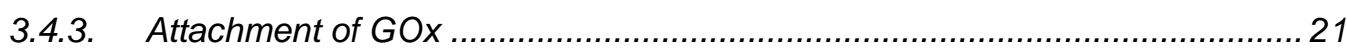

3.4.4. Electrochemical glucose sensing ........................................................ 23

$\begin{array}{lll}\text { 4. Conclusions } & 27\end{array}$

$\begin{array}{lll}\text { 5. } & \text { Future works } & 27\end{array}$

$\begin{array}{lr}\text { References } & 28\end{array}$ 


\section{CHAPTER 1}

\section{Introduction}

This chapter presents an introduction to nanoscience, nanotechnology, and nanomaterial. It also presents $\mathrm{ZnO}$ and its potential for biomolecule sensing applications. Finally, the objectives of this thesis work will be listed in the end of this chapter.

\subsection{Nanoscience and nanotechnology}

In 1959, Nobel laureate Richard P. Feynman gave a science lecture known as “There's Plenty of Room at the Bottom" [1]. It was a technological vision of a new field of physics that would come to be credited the beginning of nanotechnology. In the lecture, Feynman addressed the challenges of manipulating and controlling matter at the scale of a nanometer (one billionth of a meter or $10^{-9} \mathrm{~m}$ ) with atomic precision, and asked "Why cannot we write the entire 24 volumes of the Encyclopedia Britannica on the head of a pin?" [1]. Since then, many discoveries and inventions have led to the possibility to engrave nanoscale patterns on a solid surface. One of the first examples was the opening page of Charles Dicken's novel $A$ Tale of Two Cities written on the head of a pin with each letter only 50 atoms wide [2]. The most difficult part was to find the engraved text afterwards, but once found, there was indeed plenty of room at the bottom. In 1986, the word "nanotechnology" was used for the first time in the book Engines of Creation (1986) by K. Eric Drexler [3].

Today, nanotechnology is essentially any technology with the ability to manipulate and control individual atoms and molecules at the nanoscale. It is the application of nanoscience, which is the study of nanoscale materials. By conventional definition, a nanomaterial is defined as a material in the length scale less than $100 \mathrm{~nm}$ in one or more dimensions, and exhibits unique properties, functionality and phenomena compared to the bulk form of that material [4-6].

One of the consequences of reducing the size of a nanomaterial from 3D (bulk) and towards 2D (thin film), 1D (nanowire), and OD (quantum dot) is the quantum confinement effect. This effect relates to the spatial confinement of electrons in a nanomaterial, mainly in semiconductors, when the size is close to or even smaller than the Bohr exciton radius. In such condition, electrons are confined in all directions and exist in quantized energy levels rather than continuous, which leads to a size-dependent band gap that expands with reduced 
particle size [5-6]. Other important characteristics in nanoscale are the collective surface area and surface-to-volume ratio that increase with reduced particle size [5], which is attracted in biosensor applications for immobilization of bioreceptors.

With the recent advances in miniaturization, numerous nanostructured metal-oxides have been investigated for the possibility to engineer biosensor devices that can be used in vivo or non-invasively for continuous monitoring of blood glucose. Nanostructured metal-oxides can be synthesized in a wide range of morphologies in the length scale that is comparable to biochemical species, and therefore offer enhanced sensing characteristics.

\subsection{Zinc oxide}

\subsubsection{Development}

Zinc oxide $(\mathrm{ZnO})$ is one of the metal-oxides, which has been extensively studied. It is a mineral that occurs naturally in nature as zincite [7], and has traditionally been used as color pigment since it was introduced under the name "Chinese white" in 1834 by Winsor and Newton [8]. Today, $\mathrm{ZnO}$ is more well-known as a semiconductor material that has been studied by the scientific community since the early days of electronics. The earliest research on $\mathrm{ZnO}$ focused mainly on bulk samples and can be dated back to the 1930s. The research at that time covered studies of the thermodynamics [9], lattice parameters [10], and point defects [11].

The interest in $\mathrm{ZnO}$ started to increase at the end of the 1960s and the first noticeable research peak occurred in the 1980s Figure 1. The research topic at this time was mainly focused on bulk samples covering growth [12]. In addition, there are also a few studies on doping and electrical transport, band structure and excitons, and optical properties [13]. $\mathrm{ZnO}$ displays unique properties that attract interests as transparent conductive films [14], and hetero p-n junctions for optoelectronic applications [15]. It is known that undoped $\mathrm{ZnO}$ is naturally an n-type semiconductor, possibly due to intrinsic defects (zinc interstitials/oxygen vacancies). On the other hand, p-type conductivity is an important prerequisite for most semiconductor device applications. However, obtaining p-type conductivity in $\mathrm{ZnO}$ is a major challenge and has not yet been achieved. Hence, the interest in $\mathrm{ZnO}$ reached a plateau, partly due to the challenges of achieving stable and reproducible p-type $\mathrm{ZnO}$, and partly due to the coming era of nanotechnology. The interest was shifted to structures of reduced dimensionality and growth of nanostructures, such as quantum wells, wires and dots, which were mainly based on semiconductor compounds from other groups, such as III-V [12]. 
In 1986, pioneering work of the low-temperature buffer layer technology enabled breakthroughs in the growth of high-quality semiconductor crystals, which led to the realization of p-type conductivity in for example, GaN, and consequently the invention of the world's first GaN p-n junction blue/ultraviolet LED [16]. As a result of the improvements of growth technologies for the fabrication of high-quality single crystals, $\mathrm{ZnO}$ gained renewed interest and the number of published paper increased more significantly than in the past, as shown in Figure 1.

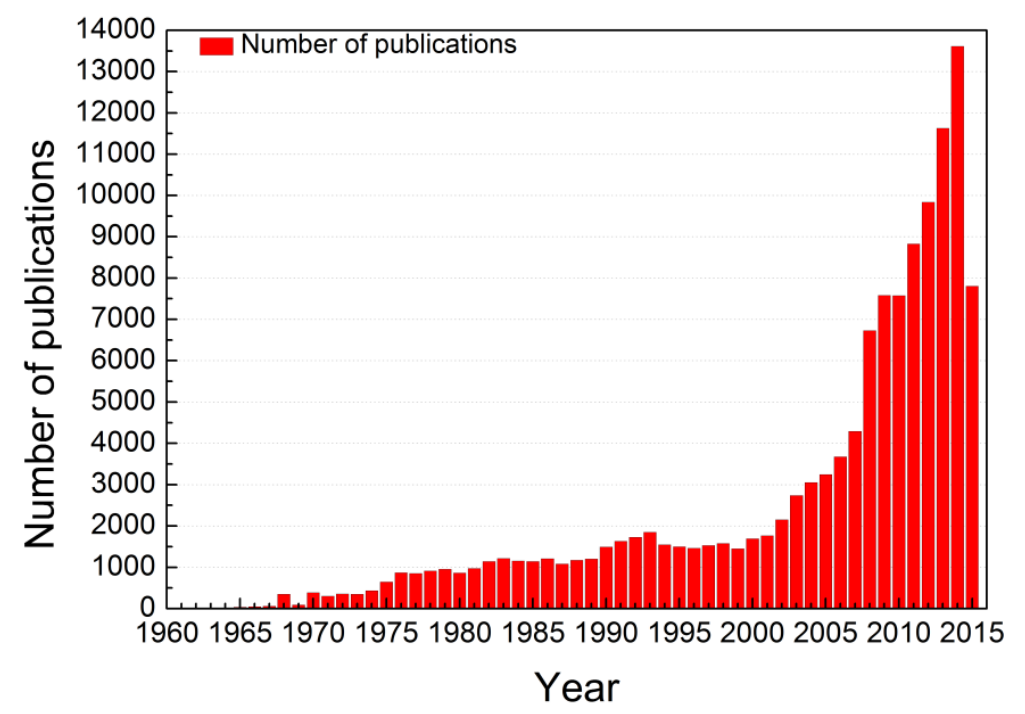

Figure 1. Number of publications based on ZnO from 1950 to present. SOURCE: Web of Science.

\subsubsection{Fundamental properties}

$\mathrm{ZnO}$ is considered one of the most versatile n-type semiconductor materials in terms of morphology and properties. It has a direct and wide band gap of $3.37 \mathrm{eV}$ in the near-UV spectral region at room temperature [12], which is comparable to that of $\mathrm{GaN}(3.39 \mathrm{eV})$ and makes $\mathrm{ZnO}$ a potential candidate for optoelectronic applications in the blue/UV region [17]. $\mathrm{ZnO}$ also exhibits an exciton binding energy of $60 \mathrm{meV}$, which is the highest among II-VI compunds [12], and allows stable excitonic emission at room temperature. Another important property is the electron mobility, which is dependent on band gap, growth technique and temperature. To date, Look et al. have reported a room-temperature electron mobility of $205 \mathrm{~cm}^{2} \mathrm{~V}^{-1} \mathrm{~s}^{-1}$ for bulk forms [18], while Ohtomo and Tsukazaki reported 440 $\mathrm{cm}^{2} \mathrm{~V}^{-1} \mathrm{~s}^{-1}$ for thin films [19].

$\mathrm{ZnO}$ are presented in three different crystal structures, wurtzite, zinc blende and rocksalt, of which the thermodynamically stable structure under ambient conditions is the wurtzite [20]. $\mathrm{ZnO}$ wurtzite has a hexagonal unit cell with lattice parameters $a=b=3.249 \AA$ and 
$c=5.206 \AA$, which yields a ratio of $c / a=1.602$ [6]. The hexagonal $\mathrm{ZnO}$ wurtzite structure is composed of alternating stacking planes of $\mathrm{Zn}$ and $\mathrm{O}$ atoms in a tetrahedral coordination along the hexagonal c-axis, as shown in Figure 2. The tetrahedral coordination creates a polar symmetry along the c-axis, which is the origin to its piezoelectricity [21], and plays an important role in crystal growth and micromechanical systems including sensors, actuators, and transducers [22].
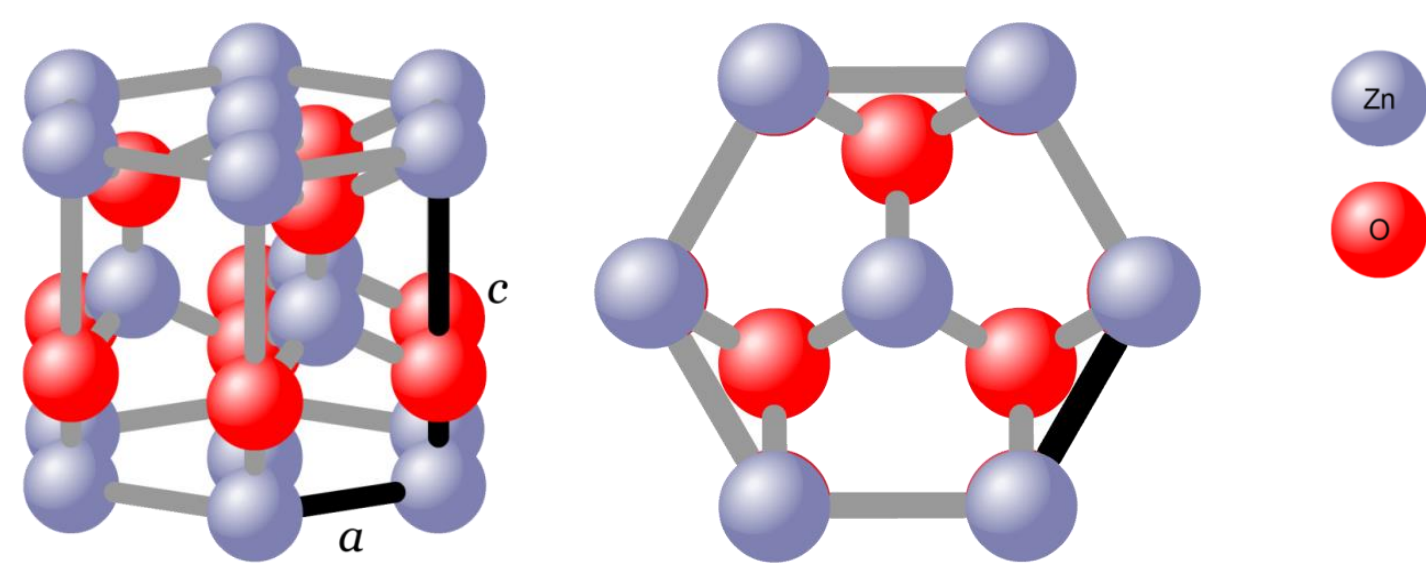

Figure 2. Hexagonal $\mathrm{ZnO}$ wurtzite structure from side view and top view, with the elements $\mathrm{Zn}$ (purple) and $\mathrm{O}$ (red). The 3D structures were drawn in 3D Crystal Viewer.

Previously reported glucose biosensors have claimed biocompatibility and non-toxicity in $\mathrm{ZnO}$ nanoparticles without providing detailed evaluation or evidence for such statements. The mechanism of cytotoxicity of $\mathrm{ZnO}$ nanoparticles in vivo or in vitro is not clear, and detailed studies are needed. For instance, $\mathrm{ZnO}$ nanoparticles are used in cancer and antibacteria treatment studies. It is demonstrated that $\mathrm{ZnO}$ nanoparticles have selective toxicity to Jurkat cancer cells [23], Escherichia coli and Staphylococcus aureus bacteria cells [24], while minimal or no effect on normal human T-cells. However, this is only true for $\mathrm{ZnO}$ nanoparticles at $0.5 \mathrm{mM}$ concentration and lower. At higher concentrations, even the normal human T-cells start to lose cell viability. The findings indicated that the cytotoxicity of $\mathrm{ZnO}$ nanoparticles is cell-specific, as well as concentration-dependent, and thus only relatively biocompatible. Studies also demonstrate that the cytotoxicity is more pronounced at higher concentrations with smaller particle size, especially in the size range of 4-20 nm [25]. Therefore, the synthesis technique to obtain $\mathrm{ZnO}$ nanoparticles plays an important role in the development of glucose biosensors that can be used in vivo. 


\subsubsection{Synthesis}

$\mathrm{ZnO}$ is possibly the semiconductor material with the richest variety of low-dimensional (2D, 1D, and 0D) nanostructures including nanocombs [26], nanorods [27], nanowires [28], nanotubes [29], and quantum dots [30]. The various nanostructures have different functional properties than bulk form, which depend on size, structure and morphological characteristics. The synthesis methods of $\mathrm{ZnO}$ nanostructures can be classified in vaporphase and solution-phase synthesis.

Vapor-phase synthesis takes place in closed systems with controlled environment and is typically carried out under high temperatures, usually 500-1500 ${ }^{\circ} \mathrm{C}$ [31]. Typical vaporphase syntheses include chemical vapor deposition (CVD), metal organic chemical vapor deposition (MOCVD), and vapor liquid solid growth (VLS). The advantage of vapor-phase synthesis is precise control of thickness, composition, and morphology. However, the vapor-phase synthesis often requires high temperature and vacuum environment with low yield, leading to a high fabrication cost.

On the other hand, solution-phase synthesis offers a wider range of cost-effective approaches, flexibility in terms of environment, and scalability. The solution-phase reactions typically occur at temperatures below $500{ }^{\circ} \mathrm{C}$ [32], which allow greater choice of substrates from organic to inorganic or even substrate-free. However, the solution-phase syntheses often involve complex chemicals and are time-consuming, typically spanning 212 hours.

By controlling the parameters during the synthesis process, $\mathrm{ZnO}$ can be synthesized in a broad variety of morphologies, such as nanotetrapods [33], nanotubes [34], or nanowires [35]. Among these nanostructures, the nanotetrapods have attracted significant attention due to their unique morphology consisting of four legs connected together in a tetrahedral symmetry. Compared to conventional nanostructures, the nanotetrapods have the advantage of multiple electron transfer paths and high aspect ratio, leading to biocompatibility and enhanced photocatalytic activity [36]. Moreover, the unique morphology allows the assembly of 3D interconnected networks, which can bridge gaps or even build up into larger architectures. However, few have reported the synthesis of nanotetrapods via solution-phase, or vapor-phase without the requirement of closed systems.

Recently, it is reported that nano-microstructured $\mathrm{ZnO}$ tetrapods ( $\mathrm{ZnO}-\mathrm{Ts}$ ) can be synthesized by flame transport synthesis (FTS), which is a vapor-phase synthesis that was first introduced by Adelung et al. [37]. The FTS is a simple and rapid up-scalable synthesis 
technique in an open environment, and believed to be the fastest reported yet [38]. It has the advantages of fast and versatile synthesis process (3-5 s), scalability (up to kilograms), and direct assembly of 3D interconnected nanostructures (on chip or device). In contrast to conventional flame synthesis methods that require precursor materials in gas-phase, FTS uses precursor metal microparticles. Compared to the conventional nanotetrapods mentioned above, the produced nano-microstructured $\mathrm{ZnO}$-Ts used in this work can reduce cellular uptake while still exhibiting specific nanomaterial properties due to the nanoscale tips. The $\mathrm{ZnO}-\mathrm{Ts}$ can be a potential candidate for biomolecule sensing applications.

\subsubsection{Applications}

The direct wide band gap, the large exciton binding energy, as well as the availability of high-quality $\mathrm{ZnO}$ bulk single crystal open up possibilities for a wide range of applications and devices. $\mathrm{ZnO}$ is considered as an alternative or a complement to $\mathrm{GaN}$ for optoelectronic applications. On the other hand, $\mathrm{ZnO}$ is also widely investigated for dye-sensitized solar cells [39], photodetectors [40], and UV lasers [41].

Moreover, owing to its photosensitivity, chemical stability, and the large band gap, $\mathrm{ZnO}$ is also investigated as photocatalyst for degradation of organic compounds and environmental pollutants. $\mathrm{ZnO}$ is comparable to $\mathrm{TiO}_{2}$, which together are the two most extensively investigated photocatalysts. However, a recent comparative study confirmed $\mathrm{ZnO}$ as the more active photocatalyst due to its ability to absorb a larger fraction of the solar spectrum [42]. Due to its photocatalytic activity, $\mathrm{ZnO}$ can also be used as a catalyst similarly to various enzymes in enzymatic glucose biosensors [43].

Furthermore, the non-centrosymmetric hexagonal wurtzite crystal structure makes $\mathrm{ZnO}$ piezoelectric, which is the property to generate electrical charge in response to mechanical stress. The piezoelectric effect makes $\mathrm{ZnO}$ attracted in the field of microelectromechanical systems (MEMS), such as electric nanogenerators [44]. At the nanoscale, nanogenerators could potentially harvest the mechanical energy from the human body (blood flow, heartbeat, and muscle stretching), which could lead to the realization of implantable biosensor devices for sustainable and continuous monitoring of blood glucose.

In the past decades, $\mathrm{ZnO}$ has been extensively investigated for the development of electrochemical biosensors for glucose sensing. The high isoelectric point (IEP) of $\mathrm{ZnO}$ (IEP=9.5), makes the semiconductor material an suitable supporting matrix for attachments of low IEP enzymes, such as GOx (IEP=4.2) by electrostatic interaction [29]. 


\subsection{Glucose biosensors}

\subsubsection{State of the art}

Diabetes mellitus is a growing public health problem worldwide and the number of people with diabetes is estimated at 171 million in 2000 and is projected to rise up to 366 million in 2030 [45]. Diabetes is characterized by insufficient insulin production (type I) in the pancreas or insulin resistance (type II) when the human body cannot utilize the produced insulin. Due to the complications of diabetes, the body is unable to properly regulate the glucose levels in the bloodstream within a normal range of 4-7 $\mathrm{mM}(80-120$ $\mathrm{mg} / \mathrm{dL}$ ) [46]. People with untreated diabetes conditions have increased risk of developing fatal diseases that affect the heart, kidney, or nerves. Glucose is the primary source of energy for cells in the body and is directly dependent on the insulin for transportation into the cells. Therefore, glucose is utilized for diagnosis and monitoring of diabetes. Various glucose biosensors have been investigated and developed in the past five decades for this purpose. Based on the transduction process, glucose biosensors are classified as amperometric, calorimetric or optical. Currently, the electrochemical glucose biosensor based on the amperometric transduction process is the most promising biosensor due to the use of glucose oxidase (GOx), which has selectivity to $\beta$-D-glucose. GOx is a homodimeric enzyme consisting of two equal subunits with a total molecular weight of $160 \mathrm{kDa}$, as shown in Figure 3. Each subunit has a redox center, which contains one mole of flavin adenine dinucleotide (FAD) non-covalently embedded within the subunit. The FAD is the cofactor responsible for oxidizing glucose to gluconolactone and produce hydrogen peroxide. The blue-marked (1) and (2) shown on the flavin are the active sites where the redox reactions take place.
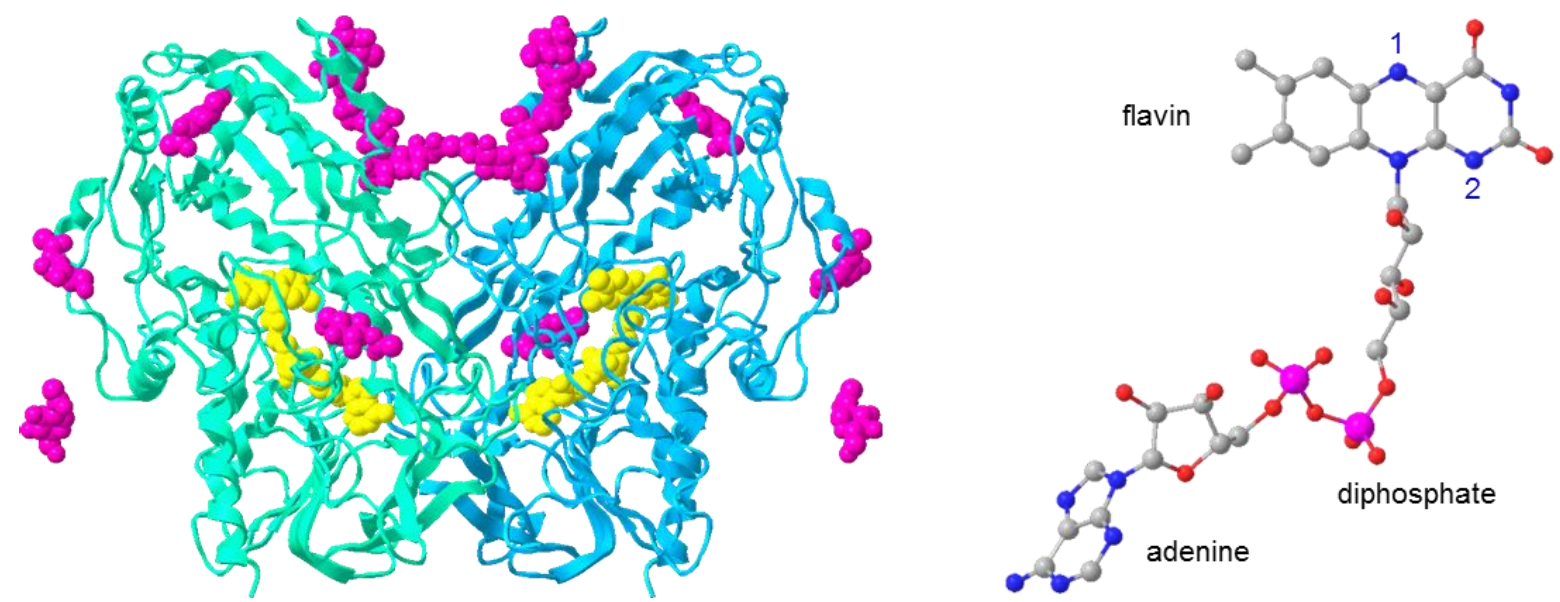

Figure 3. GOx with two FAD (yellow) and next to it is a single FAD in fully oxidized form, with elements $\mathrm{C}$ (grey), $\mathrm{O}$ (red), $\mathrm{N}$ (blue), and $\mathrm{P}$ (pink). The 3D structures were drawn in ChemBio3D Ultra. 
Three generations of the electrochemical glucose biosensor are reported, of which the third generation is currently investigated towards implantable biosensor devices for in vivo use. All three generations utilize GOx to catalyze the oxidation of glucose and the process involves two half-reactions, the reductive and the oxidative. In reductive half-reaction, FAD acts as an oxidizing agent and gains two electrons and two protons from one glucose molecule. The FAD then forms two hydrogen bonds at its active sites on the flavin and becomes reduced to $\mathrm{FADH}_{2}$, while the glucose molecule is oxidized to gluconolactone. In the oxidative half-reaction, the reduced form $\mathrm{FADH}_{2}$ is now a reducing agent and becomes reversibly oxidized to FAD in the presence of molecular oxygen, while the molecular oxygen is reduced to hydrogen peroxide. The redox process between FAD/FADH ${ }_{2}$ in GOx is reversible and capable of oxidizing glucose in the process. Therefore, GOx is widely used for the determination of glucose, since one mole of glucose is proportional to the oxygen consumed in the enzymatic oxidation. The two half-reactions are summarized in Equation 1-2, and then combined in Equation 3

$$
\begin{aligned}
\beta \text {-D-Glucose }+\mathrm{FAD} & \rightarrow \text { D-Glucono- } \delta \text {-lactone }+\mathrm{FADH}_{2} \\
\mathrm{FADH}_{2}+\mathrm{O}_{2} & \rightarrow \mathrm{FAD}+\mathrm{H}_{2} \mathrm{O}_{2} \\
\beta \text {-D-Glucose }+\mathrm{O}_{2} & \rightarrow \text { D-Glucono- } \delta \text {-lactone }+\mathrm{H}_{2} \mathrm{O}_{2}
\end{aligned}
$$

The first generation glucose biosensor was pioneered in 1962 by L. Clark and C. Lyons [47], and the underlying mechanism is simplified in Figure 4a. It is composed of an electrode with a thin layer of GOx entrapped between two membranes. The external semipermeable membrane allows diffusion of glucose towards the GOx layer, while the internal allowed diffusion of oxygen and hydrogen peroxide to the electrode contact. In the presence of glucose, oxygen is consumed by the enzymatic oxidation of glucose and the concentration oxygen that diffuses to the electrode contact is reduced. In effect, the current response to oxygen is reduced, and the reduction rate is inversely proportional to the glucose concentration. However, there are two major drawbacks, which are the electroactive interference of other species in the blood and the dependence on oxygen and the dependence on oxygen as natural electron acceptor, leading to the invention of the second generation biosensors. 
The second generation biosensors eliminated the drawbacks of the first generation design by using synthetic electron mediators for shuttling electrons, as shown in Figure $4 \mathrm{~b}$. The commonly used mediators are ferrocene derivatives, ferricyanides, and conducting organic salts. However, mediated systems over time demonstrated toxicity and difficulties to maintain the mediators near the electrode contact. All these drawbacks led to the demand for an accurate and mediator-free biosensor.

The third generation biosensors based on nanomaterials have with the recent advances in nanotechnology further improved the development of glucose biosensor. The goal of the current generation is to achieve an efficient direct electron transfer (DET) path between the redox center in GOx and the electrode contact, as shown in Figure 4c, thus eliminating synthetic mediators and errors from interfering species. Owing to the similar dimensions of nanoparticles and proteins, various nanostructures can be engineered as supporting matrix to promote the electrical contact between the redox center in GOx and the electrode contact. The reduced dimensionality and the absence of mediators are the main advantages of the third generation, which simplifies the biosensor, leading to a higher sensitivity and lower operating potential. To date, it has been reported in literature of successful DET between the GOx and the electrode. However, only few have provided evidence of such DET detections. The challenge of achieving DET at conventional electrodes has led to the investigation for various functional nanostructured materials.

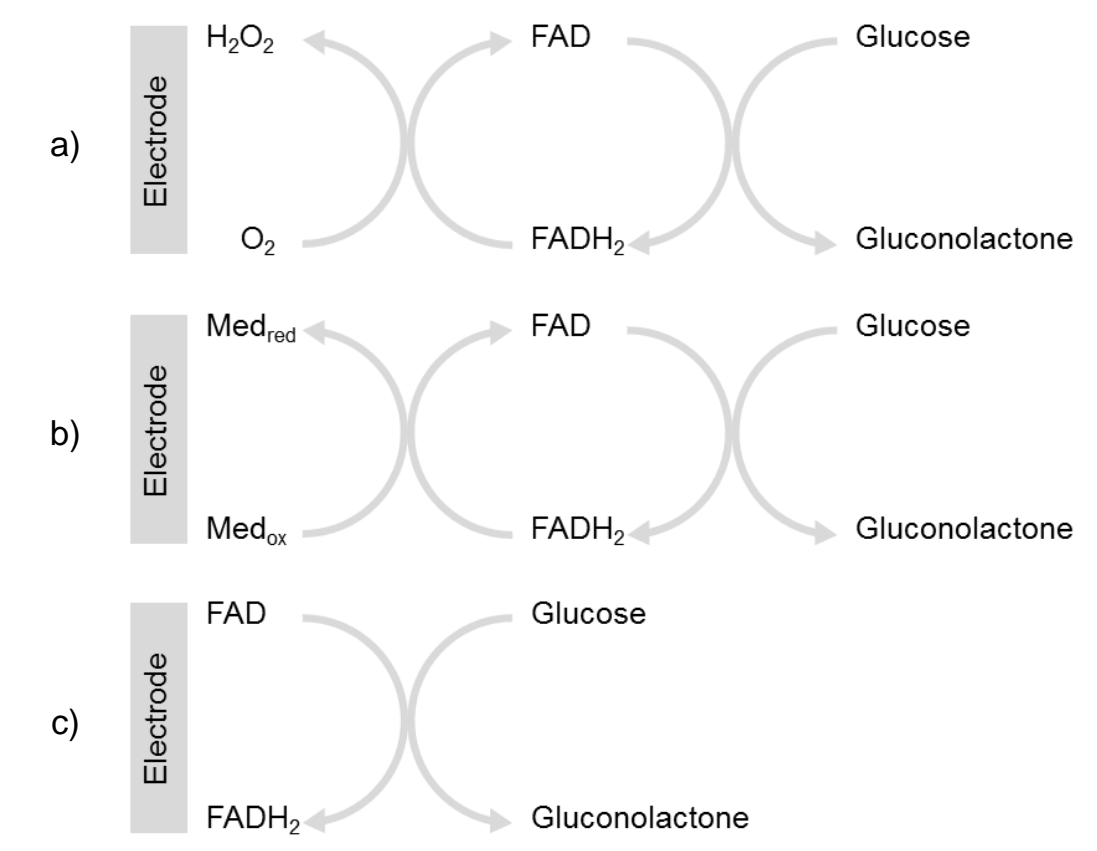

Figure 4. The evolution of the first to the third generation of electrochemical glucose biosensors. 


\subsubsection{Current challenges}

There are still challenges in the development of electrochemical glucose biosensors, mainly the immobilization of GOx on a supporting matrix while retaining the enzyme activity, and the realization of direct electron transfers between the interfaces. To optimize the glucose biosensor, various functional nanostructured materials have been investigated for a suitable matrix material during the past decade. $\mathrm{ZnO}$ is considered as one promising nanomaterial due to its rich variety of nanostructures, high electron mobility, chemical and thermal stability, and biocompatibility [48]. Recently, $\mathrm{ZnO}$ nanostructures have been reported as promising candidates, such as nanocombs [26], nanotubes [29], and nanotetrapods [33]. However, only few have reported glucose biosensors based on $\mathrm{ZnO}$ nano-microstructures.

\subsection{Objectives}

A systematic study on the structural, optical and electrochemical properties of nanomicrostructured $\mathrm{ZnO}-\mathrm{Ts}$ is reported. The large surface of $\mathrm{ZnO}$-Ts was utilized to form 3D interconnected networks as supporting matrix on a gold electrode for the attachment of GOx. For immobilization of the GOx and the ZnO-Ts architecture on the electrode, a thin film of Nafion ${ }^{\circledR}$ was over coated on the electrode surface to form a membrane. Thus, the 3D layer-by-layer $\mathrm{Au} / \mathrm{ZnO}-\mathrm{Ts} / \mathrm{GOx} / \mathrm{Nafion}$ electrode forms the active media for electrochemical glucose sensing. The glucose sensing was studied by cyclic voltammetry (CV) to confirm the potential of the $\mathrm{ZnO}$-Ts for biomolecule sensing applications and the enhancement of the sensitivity. 


\section{CHAPTER 2}

\section{Experimental details}

This chapter presents the experimental details involved in this thesis work including materials and reagents, assembly of $3 D$ layer-by-layer $\mathrm{ZnO}$-Ts architecture with GOx and Nafion ${ }^{\circledR}$ for electrochemical glucose sensing, and characterization techniques.

\subsection{Reagents and materials}

Zinc oxide tetrapods (ZnO-Ts) samples were received from Prof. Rainer Adelung's group, Kiel University in Germany. Glucose oxidase (GOx, E.C.1.1.3.4. from Aspergillus niger, 100U/mg), D-(+)-glucose (99.5\%), phosphate buffered saline tablets (PBS, $0.01 \mathrm{M}$ with $\mathrm{pH}$ 7.4), and Nafion ${ }^{\circledR}(5$ wt.\%) were purchased from Sigma-Aldrich. Hydrogen peroxide $\left(\mathrm{H}_{2} \mathrm{O}_{2}, 31 \%\right)$ was purchased from BASF.

\subsection{ZnO tetrapods for electrochemical glucose sensing}

The assembly of $\mathrm{Au} / \mathrm{ZnO}-\mathrm{Ts} / \mathrm{GOx} / \mathrm{Nafion}$ electrode involved fabrication of gold-coated substrates, attachment of GOx on $\mathrm{ZnO}-\mathrm{Ts}$, and over coating of Nafion ${ }^{\circledR}$ for immobilization. The final 3D layer-by-layer $\mathrm{Au} / \mathrm{ZnO}-\mathrm{Ts} / \mathrm{GOx} / \mathrm{Nafion}$ electrode forms the active media for electrochemical glucose sensing.

\subsubsection{Fabrication of gold-coated substrates}

A thin film deposition tool (PAK 600 Coating System, Provac) was used to deposit layers of metals on an as-prepared 4-inch $\mathrm{Si} / \mathrm{SiO}_{2}$ wafer, where the $\mathrm{SiO}_{2}$ layer on the $\mathrm{Si}$ substrate was produced by thermal oxidation at $1200{ }^{\circ} \mathrm{C}$. Various intermediate metal layers were first deposited and lastly $100 \mathrm{~nm}$ of gold. The final composition and thickness are shown in Figure 5a. After deposition, the wafer was cut into lesser $0.5 \mathrm{~cm} \times 1.0 \mathrm{~cm}$ substrates for the assembly of the 3D layer-by-layer $\mathrm{Au} / \mathrm{ZnO}-\mathrm{Ts} / \mathrm{GOx} / \mathrm{Nafion}$ electrode, as shown in Figure 5b.

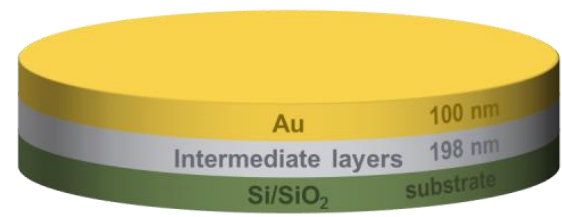

a)

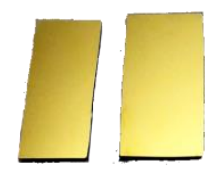

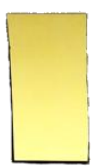

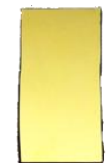

b)
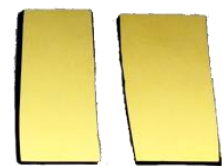

Figure 5. 3D model of a) gold-coated wafer and b) six lesser $0.5 \mathrm{~cm}$ x $1.0 \mathrm{~cm}$ gold-coated substrates. 


\subsubsection{Adhesion test of gold-coated substrates}

Plasma etching and thermal annealing were used to enhance the adhesion of the gold layer to the substrate. Plasma etching was performed in a chamber (Microwave Plasma System 300 series, TePla) for 5 min to increase the hydrophilicity. Thermal treatment was applied to improve mechanical strength and adhesion of the metal layers to the substrate. The gold-coated substrates were placed on a hotplate with the gold layer faced up, and then thermally treated at $250^{\circ} \mathrm{C}$ for 1 hour under ambient air pressure.

For the adhesion test, a scotch tape was fixed on a sample holder with the adhesive side faced up. Then, the gold-coated substrate was placed on the sample holder with the gold layer faced down. Afterwards, a weight of $100 \mathrm{~g}$ was placed on top of the configuration for $30 \mathrm{~s}$ and then the gold-coated substrate was pulled off manually from the tape. This process was repeated twice and is illustrated in Figure 6.
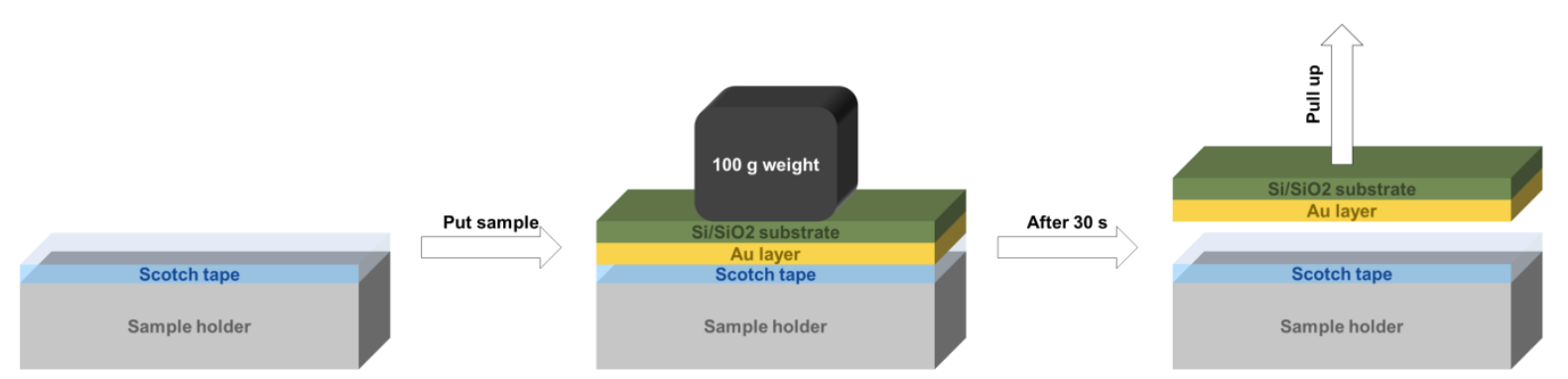

Figure 6. Schematic illustration of the pull-off test configuration and the procedure.

\subsubsection{Preparation of $\mathrm{ZnO}-\mathrm{Ts}$ suspension}

The weight-to-volume ratio of $\mathrm{ZnO}-\mathrm{Ts}$ in ethanol solution was 3:1 for all experiments. An ethanol solution was adjusted to $5 \mathrm{~mL}$, and then $15 \mathrm{mg}$ of $\mathrm{ZnO}-\mathrm{Ts}$ were immersed in the solution. The suspension of $\mathrm{ZnO}$-Ts was placed in a sonication bath for $5 \mathrm{~min}$ to disperse the agglomerates. Ethanol was used as solvent rather than DI water due to lower boiling point and increased wetting properties on gold surfaces.

\subsubsection{Preparation of glucose oxidase solution}

The weight-to-volume ratio of GOx in PBS solution was $10.0 \mathrm{mg} / \mathrm{mL}$ for all experiments. A PBS solution was prepared to $300 \mu \mathrm{L}$, and then $3.0 \mathrm{mg}$ of GOx was immersed into the solution. The GOx solution was then placed on a vortex shaker for 1 min to gently disperse the enzymes in the solution. The final solution was stored in a refrigerator at $4{ }^{\circ} \mathrm{C}$ when not used. 


\subsubsection{Assembly of the Au/ZnO-Ts/GOx/Nafion electrode}

The gold-coated substrates were immersed in a dilute solution of $\mathrm{NaOH}$ for 2 min and then rinsed with DI water to clean the surface. After drying the substrates with a nitrogen spray gun, $20 \mu \mathrm{L}$ of the $\mathrm{ZnO}$-Ts solution was drop-casted on the substrates and dried in room temperature. The drop-casting procedure was repeated 5 times until a uniform layer of $\mathrm{ZnO}$-Ts was formed on the surface. The substrates were placed on a hotplate and thermally treated at $250{ }^{\circ} \mathrm{C}$ for 10 min to evaporate the ethanol and strengthen the interface of the $\mathrm{ZnO}-\mathrm{Ts}$ and the gold surface. The obtained $\mathrm{ZnO}$-Ts architecture on the gold-coated substrate forms a functional $\mathrm{Au} / \mathrm{ZnO}$-Ts electrode. After cooling down to room temperature, $20 \mu \mathrm{L}$ of GOx solution was coated on the electrode and stored overnight to dry and establish the electrostatic interaction between the GOx and the ZnO-Ts.

After drying the GOx overnight, the electrode was rinsed with PBS to remove loosely bound GOx. Then $4 \mu \mathrm{L}$ of Nafion ${ }^{\circledR}$ solution was drop-casted on the electrode surface and stored in a refrigerator at $4{ }^{\circ} \mathrm{C}$ to dry overnight and establish the immobilization of GOx and $\mathrm{ZnO}-\mathrm{Ts}$. The final $\mathrm{Au} / \mathrm{ZnO}-\mathrm{Ts} / \mathrm{GOx} / \mathrm{Nafion}$ electrode forms the active media for electrochemical glucose sensing, as shown in Figure 7.

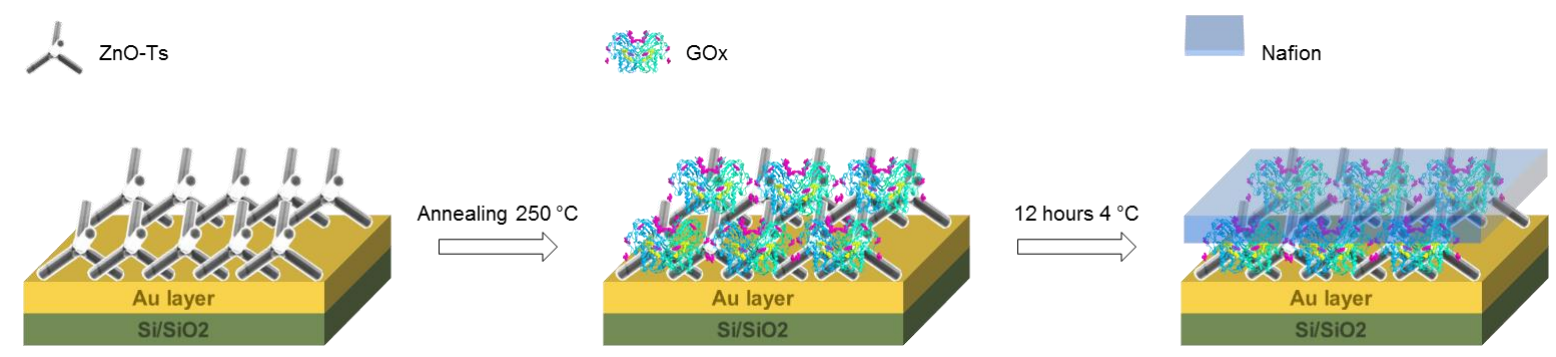

Figure 7. Preparation steps for 3D layer-by-layer Au/ZnO-Ts/GOx/Nafion electrode for electrochemical glucose sensing.

\subsection{Characterization techniques}

\subsubsection{Scanning Electron Microscopy}

A scanning electron microscope (FE-SEM: Ultra 55, ZEISS) was used for characterization of composition, size distribution, and surface morphology. SEM provides direct 3D visualization of individual objects in the nanoscale. In this work, the micrographs were produced by the in-lens detector, using an acceleration voltage of $1-3 \mathrm{kV}$, and a working distance of $5 \mathrm{~mm}$.

\subsubsection{Transmission Electron Microscopy}

A high-resolution transmission electron microscope (TEM: JEM-200F, JEOL) was used for crystallinity characterization of the $\mathrm{ZnO}$-Ts. TEM provides $2 \mathrm{D}$ information of the 
internal structures of solid samples from diffracted electrons or transmitted electrons through the sample. The micrographs were produced at an accelerating voltage of $200 \mathrm{kV}$.

\subsubsection{Fluorescence Spectroscopy}

A fluorescence spectrometer (FS: LS55, PerkinElmer) was used for optical emission analysis. The photoluminescence of $\mathrm{ZnO}$-Ts in various glucose concentration conditions was studied. In most enzymatic glucose biosensors, GOx is used as a catalyst in the enzymatic oxidation of glucose. Here, ZnO-Ts act as the catalyst similar to the GOx. The excitation wavelength used was $325 \mathrm{~nm}$, scan range from 325 to $600 \mathrm{~nm}$ at a scan rate of $100 \mathrm{~nm} / \mathrm{min}$, and slit size 15 .

\subsubsection{Stereo Microscopy}

An optical stereo microscope (SM: SMZ800, Nikon) was used in the chemistry lab to study the surfaces of gold-coated substrates. The SM provides a direct visualization and quick evaluation of samples with the naked eye. The photographs were taken by the objective lens ED Plan $0.75 x$.

\subsubsection{Fourier Transform Infrared Spectroscopy}

A fourier transform infrared spectrometer (FT-IR: VERTEX 70V, Bruker) with an A 510/Q 11 Specular Reflection Unit was used to analyze the attachment of GOx on ZnO-Ts. The FTIR spectra were obtained in reflectance mode.

\subsubsection{Cyclic Voltammetry}

A potentiostat/galvanostat (CV: EG\&G 263A, Princeton Applied Research) was used for electrochemical analysis. For the electrochemical characterizations, a conventional threeelectrode configuration was used comprising a platinum foil counter electrode (CE), a saturated $\mathrm{Ag} / \mathrm{AgCl}$ reference electrode (RE), and a gold working electrode (WE). The electrolyte used was a 0.01 M PBS of $\mathrm{pH} 7.4$ at room temperature. In potentiostatic mode, an external voltage is applied and the current response is measured. 


\section{CHAPTER 3}

\section{Results and discussion}

This chapter presents the characterization results of the $\mathrm{ZnO}$-Ts including the optical and physical properties, the attachment of GOx, and finally the performance of $3 D$ layerby-layer Au/ZnO-Ts/GOx/Nafion electrode for electrochemical glucose sensing.

\subsection{Size and morphology}

The ZnO-Ts were grown by the recently introduced synthesis technique FTS. This technique allows rapid and up-scalable synthesis of various nano-microstructures that can be deposited directly on patterned substrates. One of the interesting features of the nanomicrostructured $\mathrm{ZnO}$-Ts is the four legs connected in a tetrahedral symmetry, which allows multiple electron transfer paths and causes the $\mathrm{ZnO}$-Ts to form networks, as shown in Figure 8a. Moreover, it is observed that the legs are interconnected, shown in Figure 8b. The interconnection allows the $\mathrm{ZnO}-\mathrm{Ts}$ to bridge gaps of 2-10 $\mu \mathrm{m}$, which also contributes to the formation of larger networks and eventually 3D layer-by-layer $\mathrm{ZnO}$ architectures. Furthermore, the nano-microstructure makes the $\mathrm{ZnO}-\mathrm{Ts}$ relatively biocompatible by reducing cellular uptake while retaining nano-specific properties at the tips.

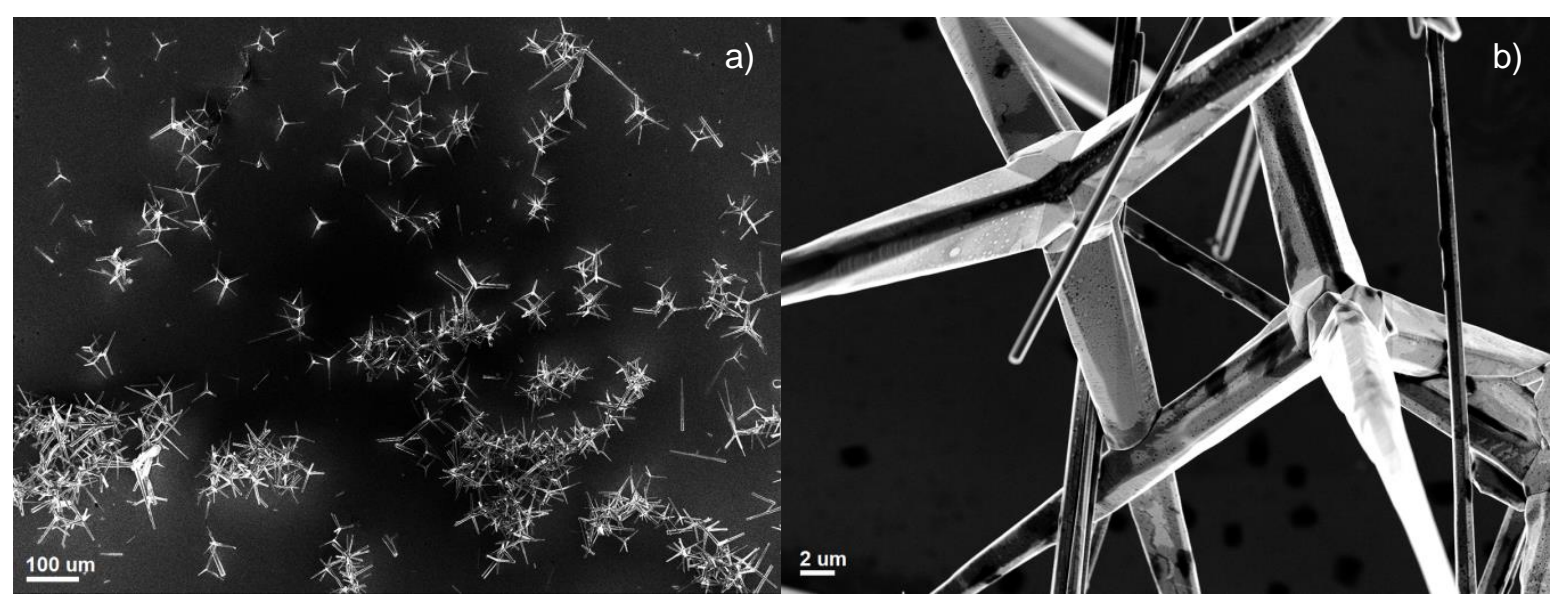

Figure 8. SEM micrographs of a) ZnO-Ts networks and b) ZnO-Ts interconnections.

The size and morphology of the ZnO-Ts were confirmed by SEM and the size distribution was obtained from at least 100 measurements in the image processing software ImageJ. SEM micrographs of 3D interconnected networks of $\mathrm{ZnO}$-Ts are shown in Figure 9a. It is observed that the $\mathrm{ZnO}-\mathrm{Ts}$ exhibit a $3 \mathrm{D}$ structure of four legs connected to a common joint, where each leg branches out from the joint pointing in a tetrahedral 
symmetry. By measuring the diameter of the legs at both the tips and the stems close to the joint of the $\mathrm{ZnO}-\mathrm{Ts}$, the average diameter of the tips and stems are confirmed to be $0.7 \mu \mathrm{m}$ and $2.2 \mu \mathrm{m}$ respectively. The size distribution of the tips and the stems is summarized in Figure 9b. It is known that the dimension of $\mathrm{ZnO}$ particles play an important role in cytotoxicity, as the toxicity is more pronounced at smaller particle size, mainly 4-20 nm [25]. In this work, the size of the $\mathrm{ZnO}-\mathrm{Ts}$ is mainly in the microscale, which is advantageous over nanoparticles in biological environment.
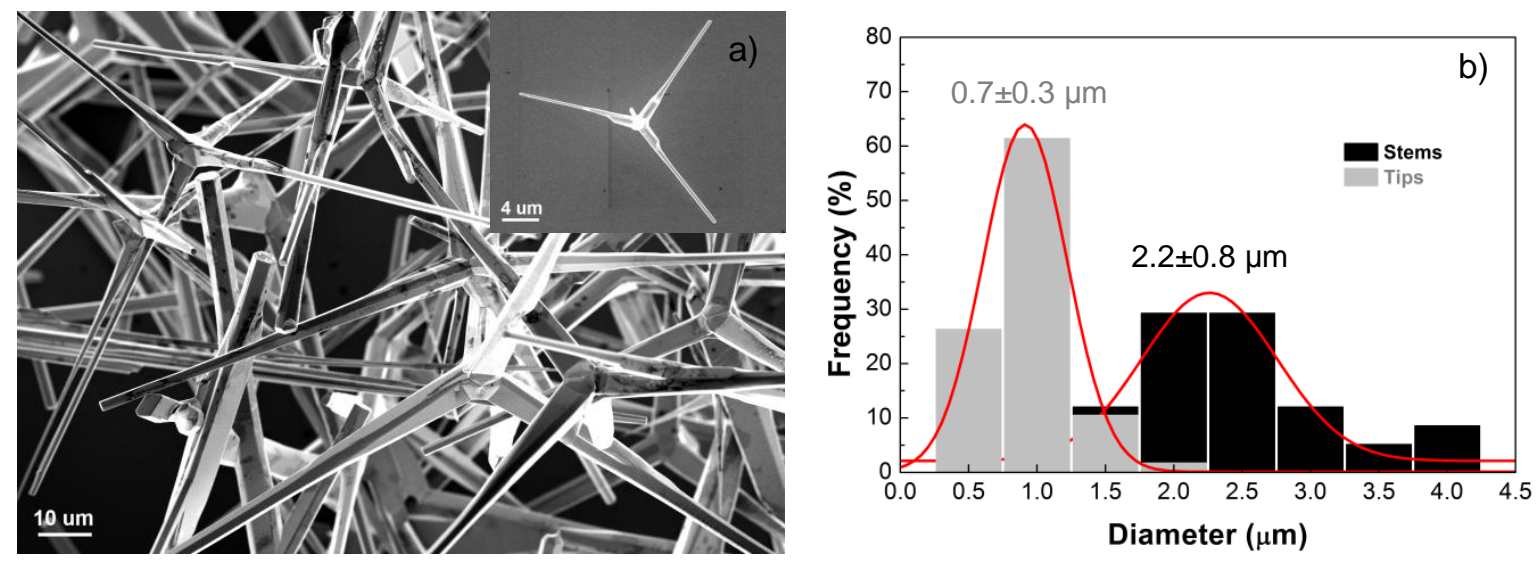

Figure 9. SEM micrograph of a) 3D interconnected networks of ZnO-Ts and b) corresponding size distribution summarized from the diameter of the tips and stems. (Inset: Single ZnO-T).

\subsection{Crystal structure}

The crystallinity of the $\mathrm{ZnO}-\mathrm{Ts}$ is confirmed by TEM in Figure 10a. The observation in TEM is consistent with that in the SEM, showing characteristics of four single crystalline legs joined together with the tips diameter in the range of $0.7 \mu \mathrm{m}$. The HR-TEM micrograph of a single ZnO-T tip reveals no visible lattice fringes due to the large size above $100 \mathrm{~nm}$. However, the periodic oriented diffraction pattern in the selected area electron diffraction (SAED) reveal the ZnO-Ts as single crystal, shown in Figure 10b.

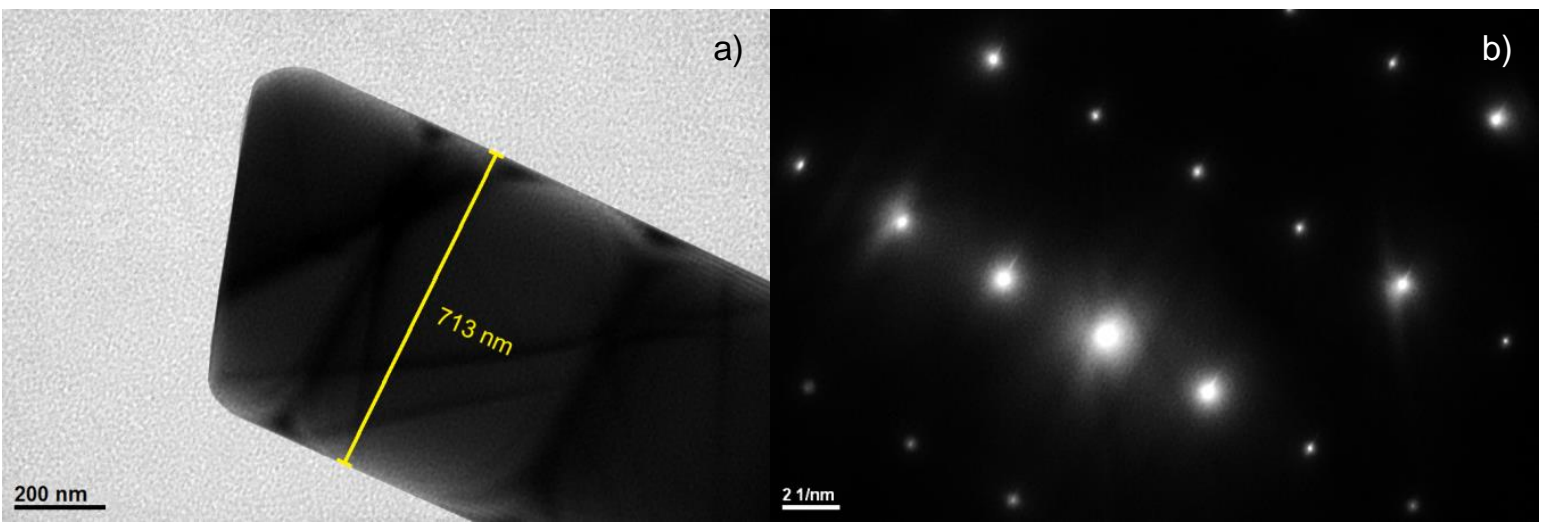

Figure 10. TEM micrograph of a) single ZnO-T tip and b) SAED pattern of that tip. 


\subsection{Optical properties}

\subsubsection{Photoluminescence}

The PL emission spectrum of $\mathrm{ZnO}$ is known to have two regions, the near-band edge emission (NBE) in the UV region and the deep-level emission (DLE) in the visible region. The origin of the NBE is from direct recombination of excitons while the DLE is suggested from radiative recombination at native intrinsic defects, such as oxygen vacancies [52]. The PL properties of ZnO-Ts were studied by fluorescence spectrometer. Two PL peaks of ZnO are observed in Figure 11a, the strong NBE peak and the broader DLE peak at $387 \mathrm{~nm}$ and $492 \mathrm{~nm}$ respectively. Morover, the PL properties of the $\mathrm{ZnO}-\mathrm{Ts}$ in various glucose concentration conditions were studied. The change in the NBE peak intensity after addition of $5 \mathrm{mM}$ glucose is normalized to the reference peak intensity of the $\mathrm{ZnO}$-Ts without glucose. It is observed that the NBE peak intensity is decreased in the presence of glucose. The proposed PL mechanism of NBE and DLE in ZnO is illustrated in Figure 11b.

As mentioned above, during UV irradiation the direct recombination of excitons yields the NBE in the form of UV-blue emission. However, when electrons in excited states get trapped at the oxygen vacancy sites on the return to ground state, the energy is released in the form of green emission, also known as DLE. Hence, the decrease of NBE peak intensity in the presence of glucose is attributed to collisional quenching by hydrogen peroxide produced from the enzymatic oxidation of glucose. The hydrogen peroxide may act as an electron acceptor similar to the oxygen vacancy, thus allowing favorable relaxation pathways, which increases the DLE peak intensity.
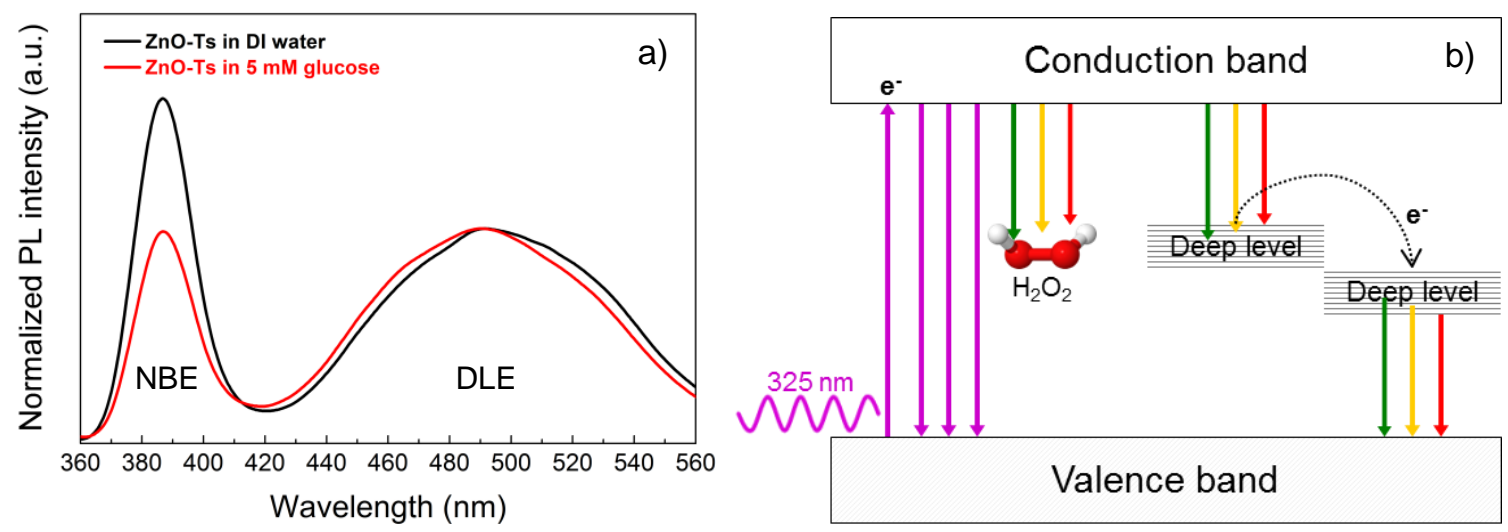

Figure 11. PL emission spectra of a) ZnO-Ts in DI water without and with $5 \mathrm{mM}$ glucose, and b) schematic diagram of the proposed PL emission and intensity quenching mechanism. 


\subsubsection{Optical glucose sensing}

Among all samples, it is found that the peak intensity and position vary in the visible region, whereas only the peak intensity varies in the UV region. Therefore in this work, the PL intensity quenching was mainly studied in the UV region. The PL spectrum of ZnO-Ts in DI water was obtained as a reference. It is observed that the NBE peak intensity is decreased in the presence of glucose and further decreased with the successive addition of glucose, as shown in Figure 12a. The change in the NBE peak intensity after the addition of glucose is normalized to the reference peak intensity of the $\mathrm{ZnO}$-Ts without glucose. It is found that the peak intensity change has a linear dependence on the glucose concentration up to $4 \mathrm{mM}$ and then approaches towards, shown in Figure 12b. The linear region barely covers the glucose level in the normal range between 4.4-6.6 $\mathrm{mM}$. The narrow linear range is suggested to be due to the surface passivation by hydrogen peroxide that is constantly accumulated from the enzymatic oxidation of glucose.
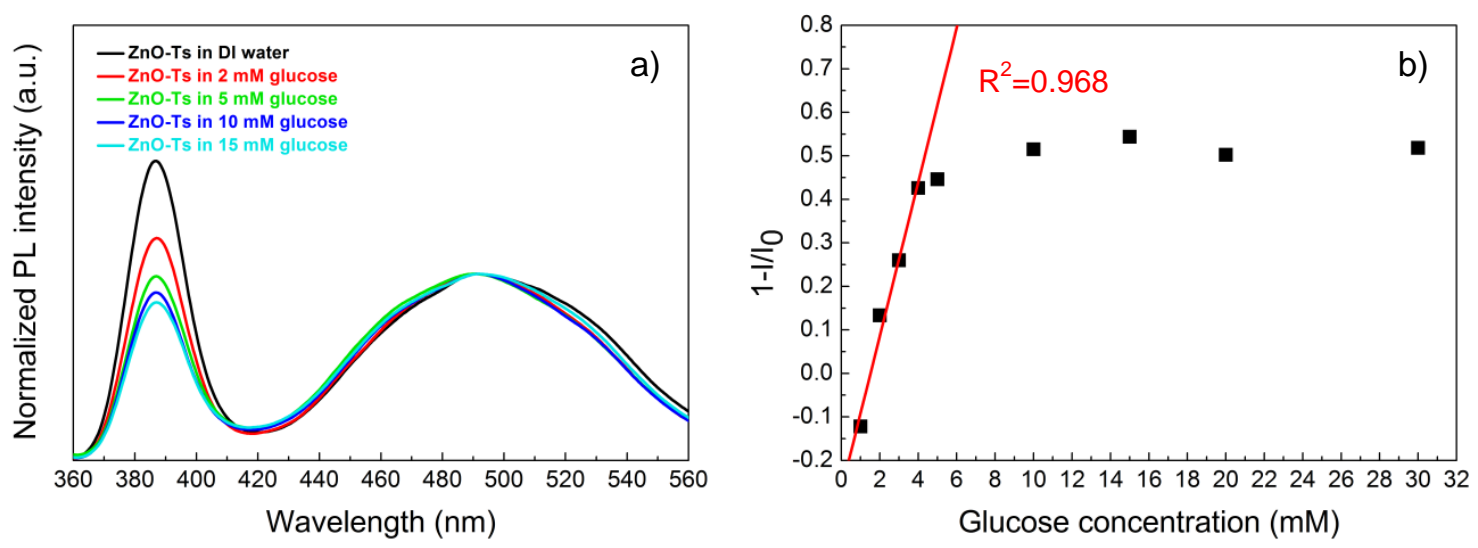

Figure 12. PL emission spectra of a) $\mathrm{ZnO}$-Ts in DI water with various glucose concentrations and b) PL intensity change of the NBE versus glucose concentration relative to that of the $\mathrm{ZnO}$-Ts without glucose.

To confirm whether the PL intensity quenching is caused by hydrogen peroxide, the PL properties of $\mathrm{ZnO}-\mathrm{Ts}$ were studied in various hydrogen peroxide concentration conditions. The hydrogen peroxide (31\%) concentration was increased successively from 5 to $30 \mathrm{mM}$ $(1.7 \mu \mathrm{L} / 5 \mathrm{mM})$ in a quartz cuvette $(3.5 \mathrm{~mL})$ with the $\mathrm{ZnO}$-Ts suspension. The PL spectrum of $\mathrm{ZnO}-\mathrm{Ts}$ in DI water was obtained as a reference. Similar as glucose, it is observed that the NBE peak intensity is decreased in the presence of hydrogen peroxide and further decreased with the successive addition, as shown in Figure 13a. The change in the NBE peak intensity after the addition of hydrogen peroxide is normalized to the reference peak intensity of the $\mathrm{ZnO}$-Ts without hydrogen peroxide. Compared to glucose, it is found that the peak intensity change has a linear dependence on the hydrogen peroxide concentration 
up to $15 \mathrm{mM}$ and then approaches towards a saturation plateau, shown Figure $13 \mathrm{~b}$. The results demonstrate that the PL intensity quenching is caused by hydrogen peroxide, which is in agreement with Kim et al. [49], and Sarangi et al. [43]. The PL intensity quenching can therefore be used to measure glucose concentration, since the quenching is proportional to the hydrogen peroxide concentration, which also is proportional to the glucose concentration. From the results, both glucose and hydrogen peroxide show PL intensity quenching. However, the change in PL intensity quenching by glucose and hydrogen peroxide are not proportional. Glucose shows a linear region up to $4 \mathrm{mM}$ and hydrogen peroxide showed up to $15 \mathrm{mM}$.

For accurate optical glucose sensing, it is desired to have $\mathrm{ZnO}$ nanoparticles of uniform size distribution rather than the nano-microstructured $\mathrm{ZnO}$-Ts used in this work, since the quenching also depends on the size of the particles. Compared to the stems of the ZnO-Ts, the energy of the valence and conduction band edges at the tips is larger by quantum confinement, and thus facilitates electron transfers more efficiently from the $\mathrm{ZnO}$-Ts to the hydrogen peroxide. The size difference affects the reliability and reproducibility of the measurements.
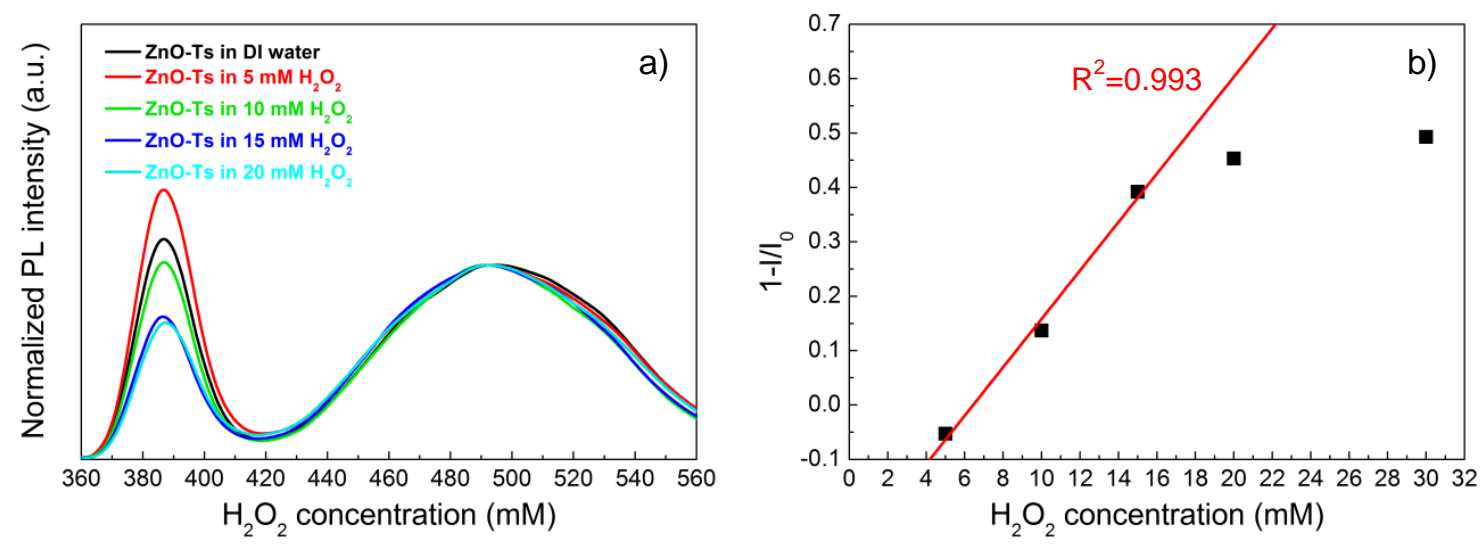

Figure 13. PL emission spectra of a) ZnO-Ts in DI water with various hydrogen peroxide concentrations and b) PL intensity change of the NBE versus hydrogen peroxide concentration relative to that of the $\mathrm{ZnO}-\mathrm{Ts}$ without hydrogen peroxide.

\subsection{Electrochemical properties}

\subsubsection{Adhesion test of gold-coated substrates}

The gold-coated substrates were washed and thermally treated on a hotplate. The gold layer adherence to the substrate without and with annealing is summarized in Table 1 . It is observed that the gold-coated substrate without annealing did not sustain the pull-off test. After the pull-offs, the gold layer was peeled off completely from the substrate and revealed 
the silica surface underneath, as shown in Table 1a. On the other hand, the gold-coated substrate with annealing sustained the pull-off test to a greater extent, and the gold layer was only partly peeled off at the borders, shown in Table 1b. It is found that annealing at elevated temperatures enhances the adherence of gold layer to the substrate.

Table 1. Pull-off test of gold-coated substrate without and with annealing treatment.

\begin{tabular}{lll}
\hline Before pull-off test & After pull-off test \\
\hline a) Without annealing & \\
b) Annealing & \\
\hline
\end{tabular}

\subsubsection{Self-assembly of ZnO-Ts}

The suspension of $5 \mathrm{mg} \mathrm{ZnO}$-Ts in $5 \mathrm{~mL}$ ethanol solution has an opaque appearance and individual $\mathrm{ZnO}-\mathrm{Ts}$ that are visible to the naked eye, as shown in Figure 14a. ZnO-Ts were drop-casted on a gold-coated substrate and then thermally treated on a hotplate to form the functional $\mathrm{Au} / \mathrm{ZnO}-\mathrm{Ts}$ electrode. The obtained $\mathrm{ZnO}$-Ts architecture on the electrode surface forms the supporting matrix for attachment of GOx. The electrode surface was studied by $\mathrm{SM}$, and the obtained photograph is shown in Figure 14b. It is observed that the ZnO-Ts are uniformly distributed in the center and partly clustered at the borders. This is due to the droplets from the drop-cast that pushed the $\mathrm{ZnO}$-Ts outwards upon landing on the surface. The distribution of $\mathrm{ZnO}$-Ts can be more uniform in future works by introducing crosslinking agents, such as (3-aminopropyl)trimethoxysilane and using spin coating method to deposit the $\mathrm{ZnO}$-Ts. 


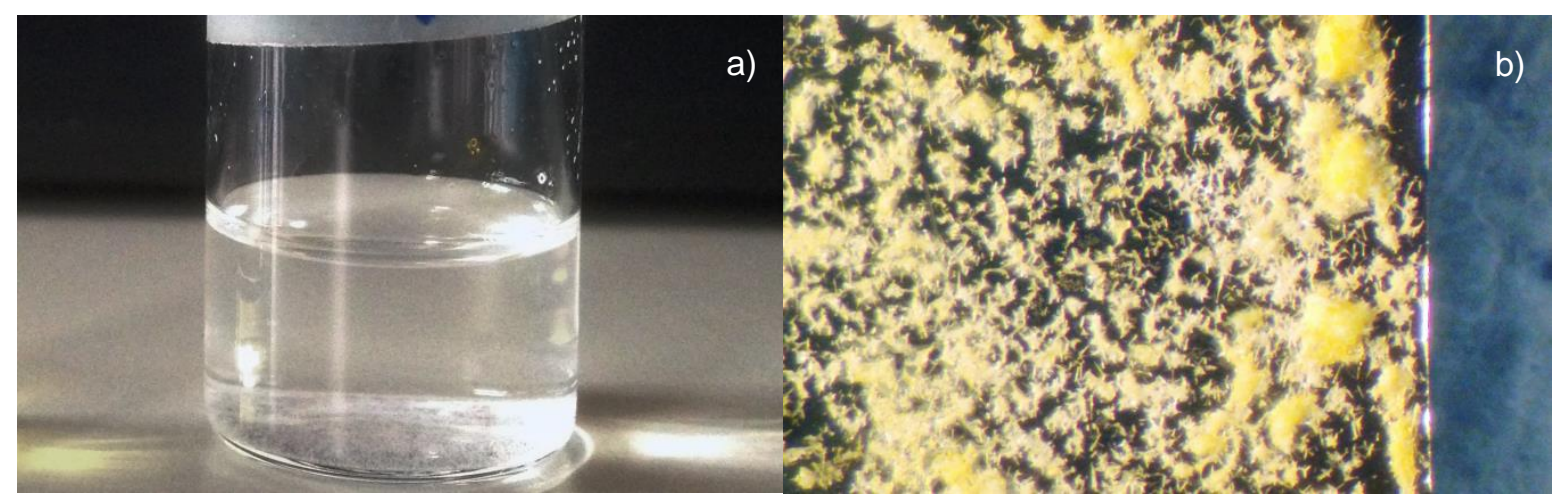

Figure 14. Photographs of a) ZnO-Ts suspension and b) 3D layer-by-layer Au/ZnO-Ts electrode.

\subsubsection{Attachment of GOx}

The GOx solution was prepared in 0.01 M PBS solution, which has a clear yellow appearance after mixing, as shown in Figure 15a. The GOx was drop-casted on the $\mathrm{Au} / \mathrm{ZnO}$-Ts electrode and dried overnight, then rinsed with PBS solution to remove loosely bound GOx. After drying, a uniform layer of Nafion ${ }^{\circledR}$ was over coated on the electrode surface by drop-casting to form the $\mathrm{Au} / \mathrm{ZnO}-\mathrm{Ts} / \mathrm{GO} / \mathrm{Nafion}$ electrode, and the final product is shown in Figure 15b. It is observed that the attached GOx (yellow) is more pronounced where the $\mathrm{ZnO}-\mathrm{Ts}$ (white) are assembled.

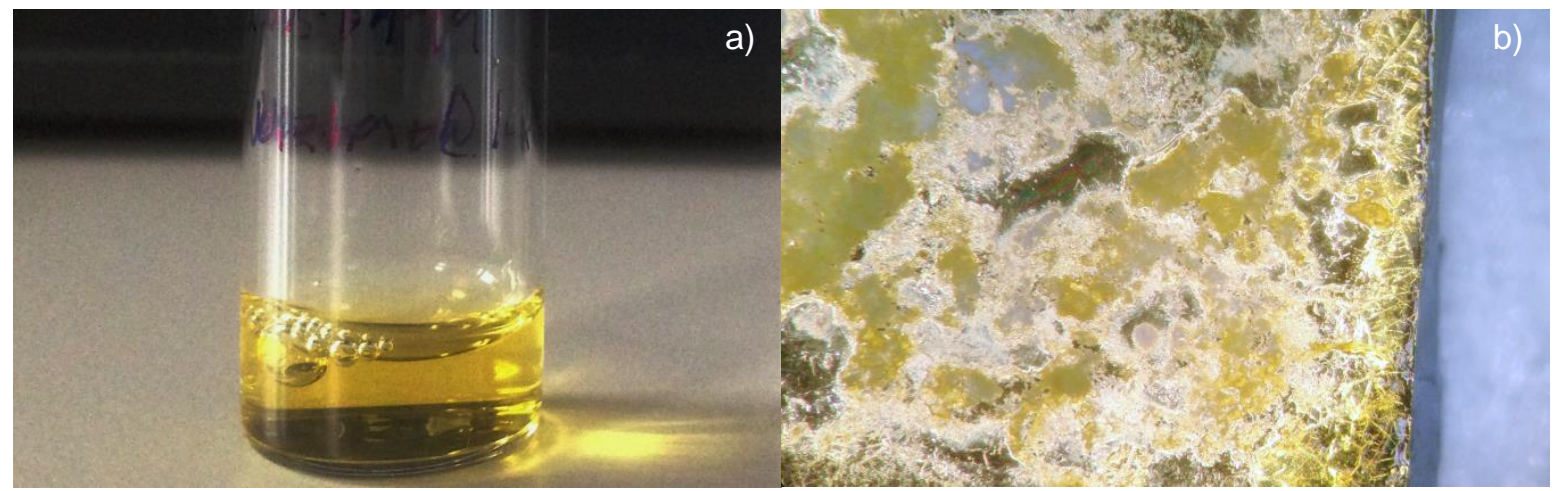

Figure 15. Photographs of a) GOx solution and b) 3D layer-by-layer Au/ZnO-Ts/GOx/Nafion electrode.

The existence of the GOx layer was confirmed by FT-IR from IR absorption without and with GOx, and the obtained reflectance spectra are shown in Figure 16b. Two bands are observed, which are associated with GOx. The amide I band at $1655 \mathrm{~cm}^{-1}$ and the amide II band at $1543 \mathrm{~cm}^{-1}$ are mainly due to $\mathrm{C}=\mathrm{O}$ stretching vibrations of peptide bonds in the protein and the latter to a combination of $\mathrm{C}-\mathrm{N}$ stretching and $\mathrm{N}-\mathrm{H}$ in-plane bending. These two bands confirm the attachment. The surface morphology of the Au/ZnO-Ts/GOx electrode was confirmed by SEM. It is observed that a uniform layer of GOx was attached on the $\mathrm{ZnO}-\mathrm{Ts}$, as shown in Figure 16a, which is consistent with the results from the FT-IR. 

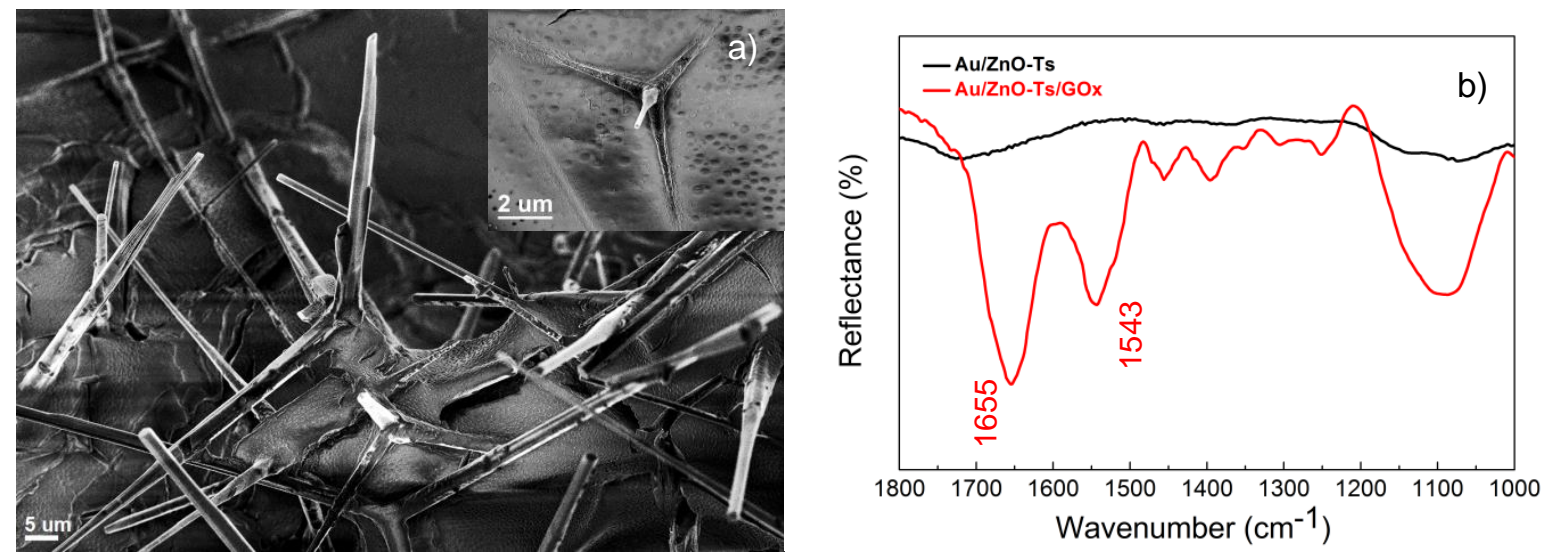

Figure 16. SEM micrograph of a) Au/ZnO-Ts/GOx electrode and b) FT-IR spectra of Au/ZnO-Ts electrode without and with GOx. (Inset: Single ZnO-T coated with GOx).

After over coated a thin layer of $\mathrm{Nafion}^{\circledR}$ on the electrode, the existence of the active layers was confirmed by FT-IR, and the obtained spectra are shown in Figure 17b. The two bands of GOx are still observed, the amide I band at $1637 \mathrm{~cm}^{-1}$ and the amide II band at $1543 \mathrm{~cm}^{-1}$. These two bands confirm that the attachment of GOx on the ZnO-Ts architecture is preserved. Additional three infrared bands are observed at $1213 \mathrm{~cm}^{-1}, 1151$ $\mathrm{cm}^{-1}$ and $1060 \mathrm{~cm}^{-1}$, which are associated with Nafion ${ }^{\circledR}$ in response to the asymmetric and symmetric $\mathrm{CF}_{2}$ stretch modes, and the latter to symmetric $\mathrm{SO}_{3}$ stretch. The surface morphology of the active layers was confirmed by SEM. It is observed that a layer of Nafion ${ }^{\circledR}$ was over coated on the ZnO-Ts, as shown in Figure 17a. The layer acts as a membrane, which prevents leakage of GOx as well as improves the stability. Nafion ${ }^{\circledR}$ was also over coated on a single $\mathrm{ZnO}-\mathrm{T}$ with GOx, which anchored the structure to the electrode surface, shown in the inset of Figure 17a. The obtained 3D layer-by-layer $\mathrm{Au} / \mathrm{ZnO}-$ Ts/GOx/Nafion electrode forms the active media for electrochemical glucose sensing.
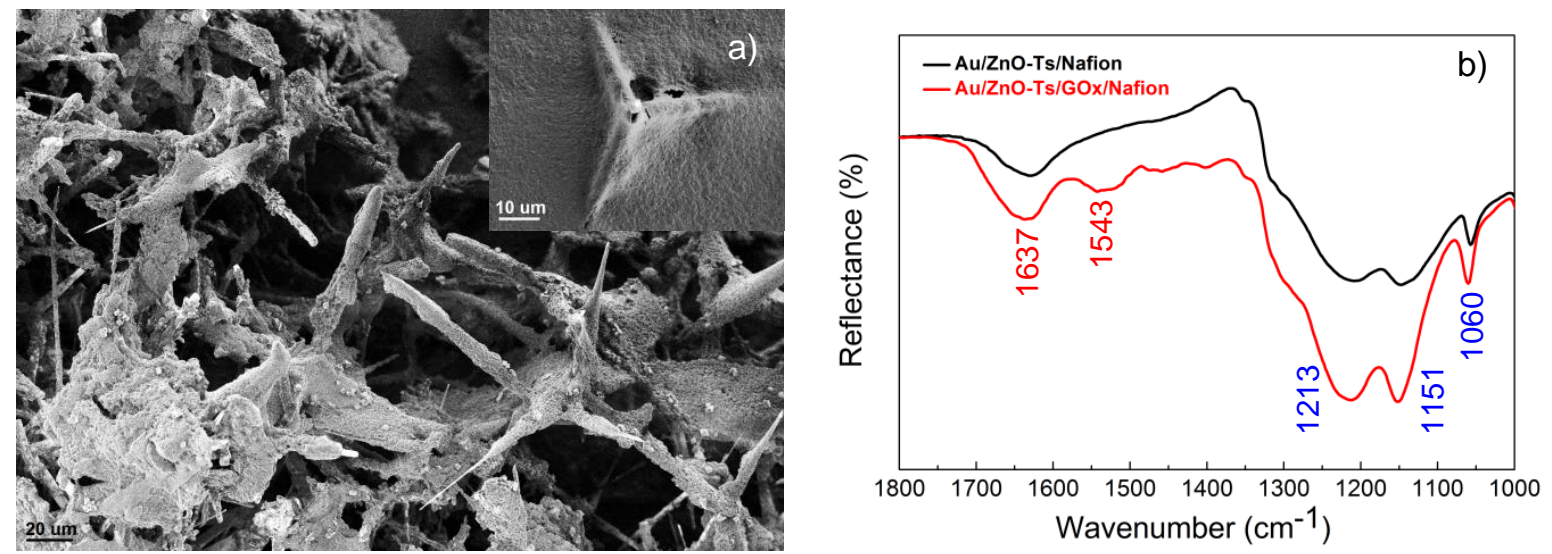

Figure 17. SEM micrograph of a) 3D layer-by-layer Au/ZnO-Ts/GOx/Nafion electrode and b) FT-IR reflectance spectra of $\mathrm{Au} / \mathrm{ZnO}-\mathrm{Ts} / \mathrm{Nafion}$ electrode without and with GOx. (Inset: Single ZnO-T coated with GOx and Nafion ${ }^{\circledR}$ ). 


\subsubsection{Electrochemical glucose sensing}

Three-electrode configuration is a widely used technique in electrochemistry for characterization of electrochemical processes and comprises of a WE coated with sample, a saturated $\mathrm{Ag} / \mathrm{AgCl} \mathrm{RE}$, and a platinum $\mathrm{CE}$. The working principle of cyclic voltammetry is illustrated in Figure 18. In potentiostatic scan mode, the potential at the WE versus the RE is ramped linearly with time, and the current is measured between the $\mathrm{CE}$ and WE. The CE, also often called the auxiliary electrode ensures that the current does not pass through the RE, since such current would cause a potential drop between the WE and the RE. The advantage of the three-electrode configuration is that the current-voltage characteristics are measured on the WE, whereas a two-electrode configuration would measure the whole electrochemical cell including the electrolyte.

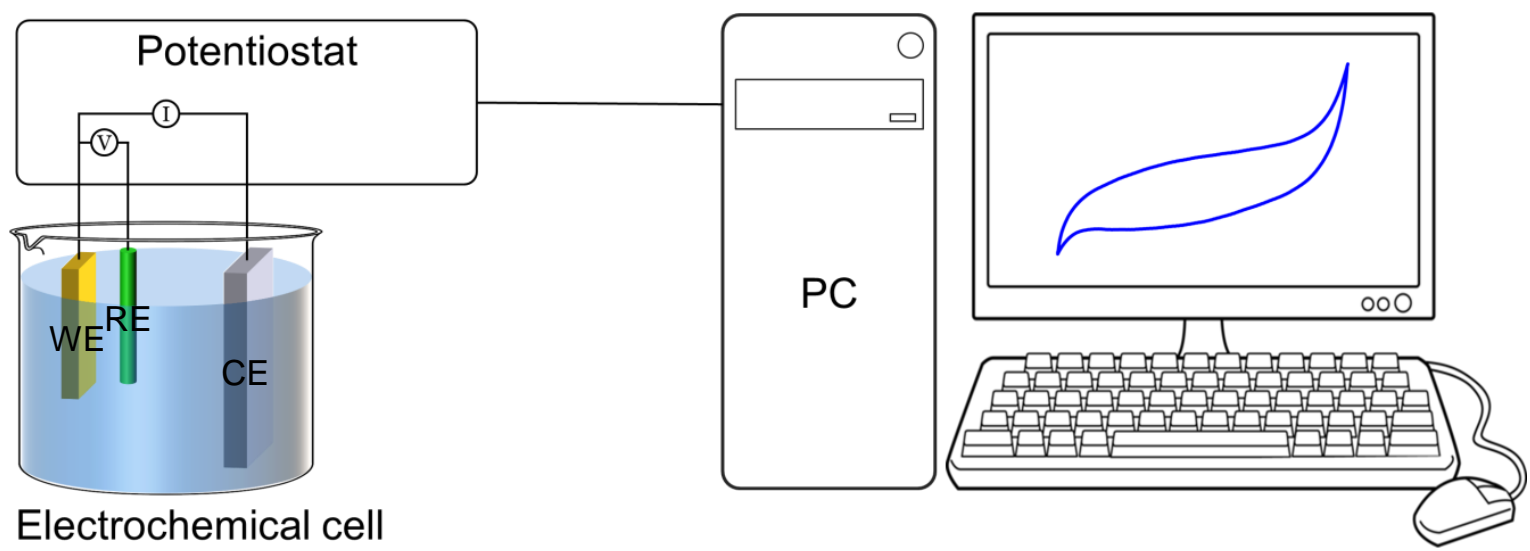

Figure 18. Schematic diagram of the electrochemical cell, potentiostat, and PC for inputs and monitoring.

Prior to current response measurements, a bare gold electrode was investigated to find the optimal working parameters, such as working region and placement of electrodes, as shown in Figure 19a. The current response of the bare gold electrode was measured in PBS from $-1.0 \mathrm{~V}$ to $1.0 \mathrm{~V}$ at the scan rate of $50 \mathrm{mV} / \mathrm{s}$. It is found that the optimal working region is between $-0.2 \mathrm{~V}$ and $0.8 \mathrm{~V}$, shown in Figure $19 \mathrm{~b}$. Moreover, the RE is placed close to the WE due to the uncompensated cell resistance effects in the electrolyte solution, which contributes to a potential shift according to Equation 4.

$$
\eta_{\text {ohmic }}=I \cdot R_{s}
$$

This expression shows that the magnitude of the potential shift, also called overpotential $\eta_{\text {ohmic }}$ depends on the current I and the cell resistance $R_{s}$. 


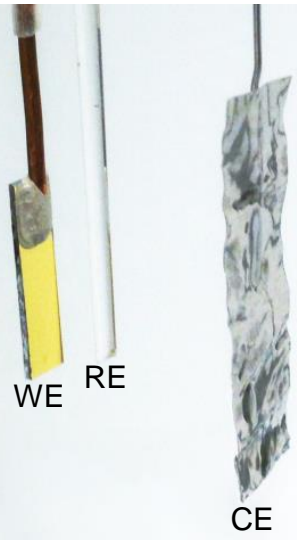

a)

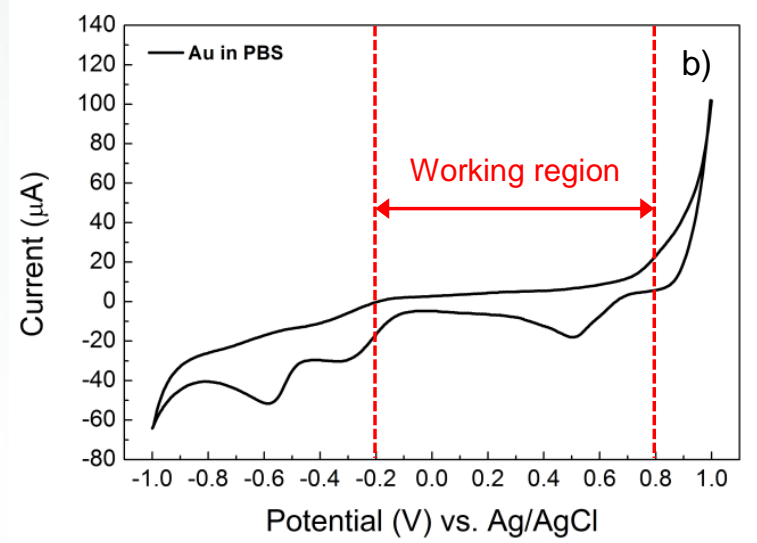

Figure 19. Photograph of a) three-electrode configuration comprising bare gold $\mathrm{WE}, \mathrm{Ag} / \mathrm{AgCl} \mathrm{RE}$ and platinum $\mathrm{CE}$, and b) cyclic voltammogram of the bare gold electrode in PBS from $-1.0 \mathrm{~V}$ to $1.0 \mathrm{~V}$ at the scan rate of $50 \mathrm{mV} / \mathrm{s}$.

The chemical stability of $\mathrm{ZnO}-\mathrm{Ts}$ on the $\mathrm{Au} / \mathrm{ZnO}-\mathrm{Ts}$ electrode was studied by measuring current response in PBS, and the obtained cyclic voltammogram is shown in Figure 20a. Compared to the electrode without $\mathrm{ZnO}$ - Ts, the current response of the electrode with $\mathrm{ZnO}$ Ts is observed to be suppressed from $0.3 \mathrm{~V}$ to $0.8 \mathrm{~V}$. In addition to the current change, an anodic peak current is also observed at $0.29 \mathrm{~V}$, which could be due to the oxidation of the $\mathrm{ZnO}-\mathrm{Ts}[33]$.

Moreover, the $\mathrm{ZnO}-\mathrm{Ts}$ were attached with GOx and over coated with $\mathrm{Nafion}^{\circledR}$ to form the $\mathrm{Au} / \mathrm{ZnO}-\mathrm{Ts} / \mathrm{GOx} / \mathrm{Nafion}$ electrode for the measurement of the current response to 5 $\mathrm{mM}$ glucose concentration in PBS, shown in Figure 20b. It is observed that the anodic current is increased while the cathodic current is suppressed from $0.32 \mathrm{~V}$ in the presence of $5 \mathrm{mM}$ glucose solution compared to that in PBS. The irreversible current response indicates the enzymatic oxidation of glucose by GOx catalysis.
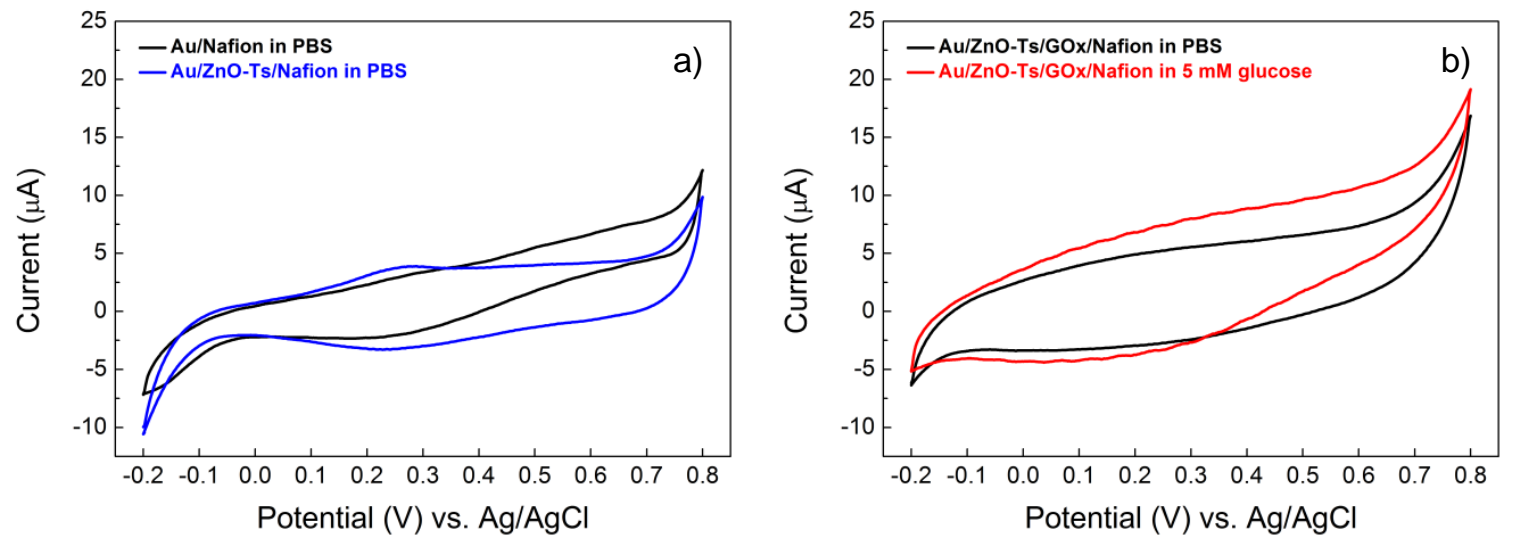

Figure 20. Cyclic voltammogram of a) Au/Nafion electrode in PBS without and with ZnO-Ts at the scan rate of $50 \mathrm{mV} / \mathrm{s}$, and b) $\mathrm{Au} / \mathrm{ZnO}-\mathrm{Ts} / \mathrm{GOx} / \mathrm{Nafion}$ electrode in PBS without and with $5 \mathrm{mM}$ glucose. 
Furthermore, the electron transfer kinetics of the electrochemical processes was studied. The current response from $\mathrm{Au} / \mathrm{ZnO}-\mathrm{Ts} / \mathrm{GOx} / \mathrm{Nafion}$ electrode to $5 \mathrm{mM}$ glucose concentration in PBS was obtained at various scan rates from $50-100 \mathrm{mV} / \mathrm{s}$, and is shown in Figure 21a. It is found that the anodic and cathodic peak currents are proportional to the square root of the scan rate, which indicates a fast electron transfer through the active layers with no diffusion limits. The anodic and cathodic peak currents versus the square root of the scan rate are compared, shown in Figure $21 \mathrm{~b}$. The linear change of peak current confirms that the generated hydrogen peroxide from the enzymatic oxidation of glucose diffused to the electrode contact and oxidized efficiently.
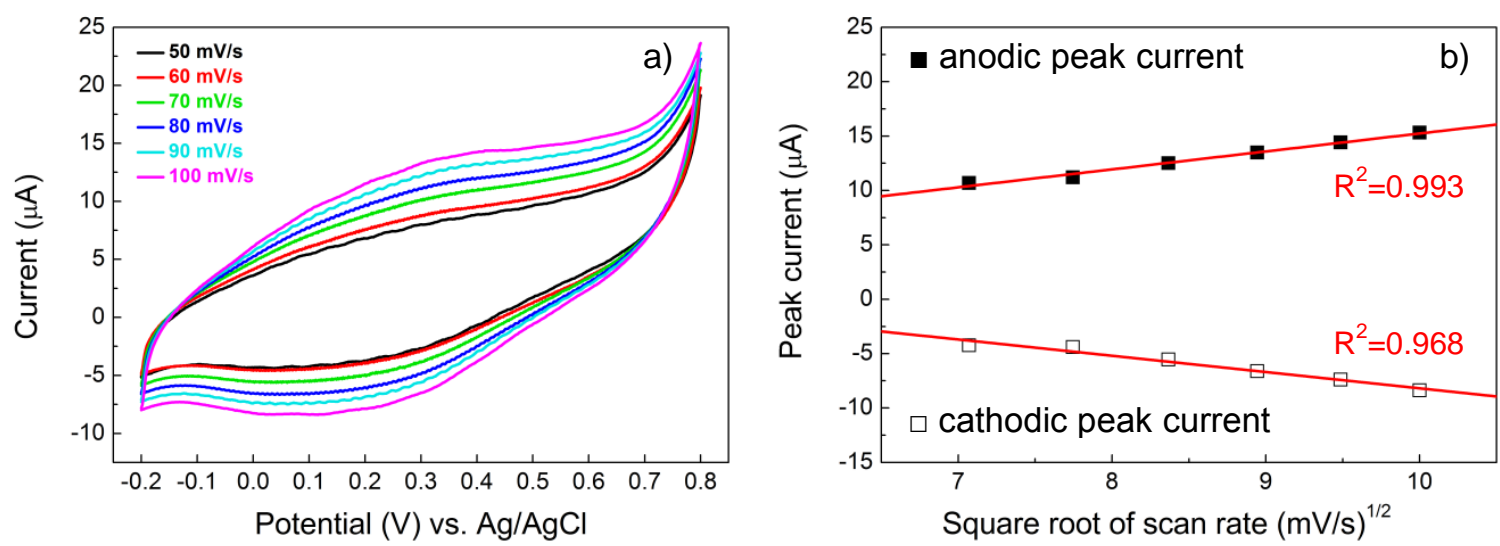

Figure 21. Cyclic voltammogram of a) Au/ZnO-Ts/GOx/Nafion electrode in PBS with $5 \mathrm{mM}$ glucose measured at scan rate from $50-100 \mathrm{mV} / \mathrm{s}$, and b) the anodic and cathodic peak current versus the square root of the scan rate.

The current response of the active layers to various glucose concentrations of $1 \mathrm{mM}$ to 40 $\mathrm{mM}$ was measured, of which the first four measurements are shown in Figure 22a. The current response of the active layers without glucose was also studied as a reference. It is observed that the current is increased from $0.4 \mathrm{~V}$ to $0.8 \mathrm{~V}$ in the presence of glucose, indicating a response of the $\mathrm{Au} / \mathrm{ZnO}-\mathrm{Ts} / \mathrm{GOx} / \mathrm{Nafion}$ electrode to glucose. The increase in current is due to enzymatic oxidation of glucose, and since the glucose reaction is not reversible, the anodic current (oxidation) is increased while the cathodic (reduction) is suppressed. The anodic current is further increased and the cathodic is suppressed with the successive addition of glucose. There is an also weak shoulder peak at $0.55 \mathrm{~V}$, which is attributed to the generated of hydrogen peroxide during the enzymatic oxidation of glucose. The anodic peak current at fixed potential $0.6 \mathrm{~V}$ is found to have a linear dependence on the glucose concentration up to $12 \mathrm{mM}$, and then approaches towards a saturation plateau, shown in Figure 22b. 

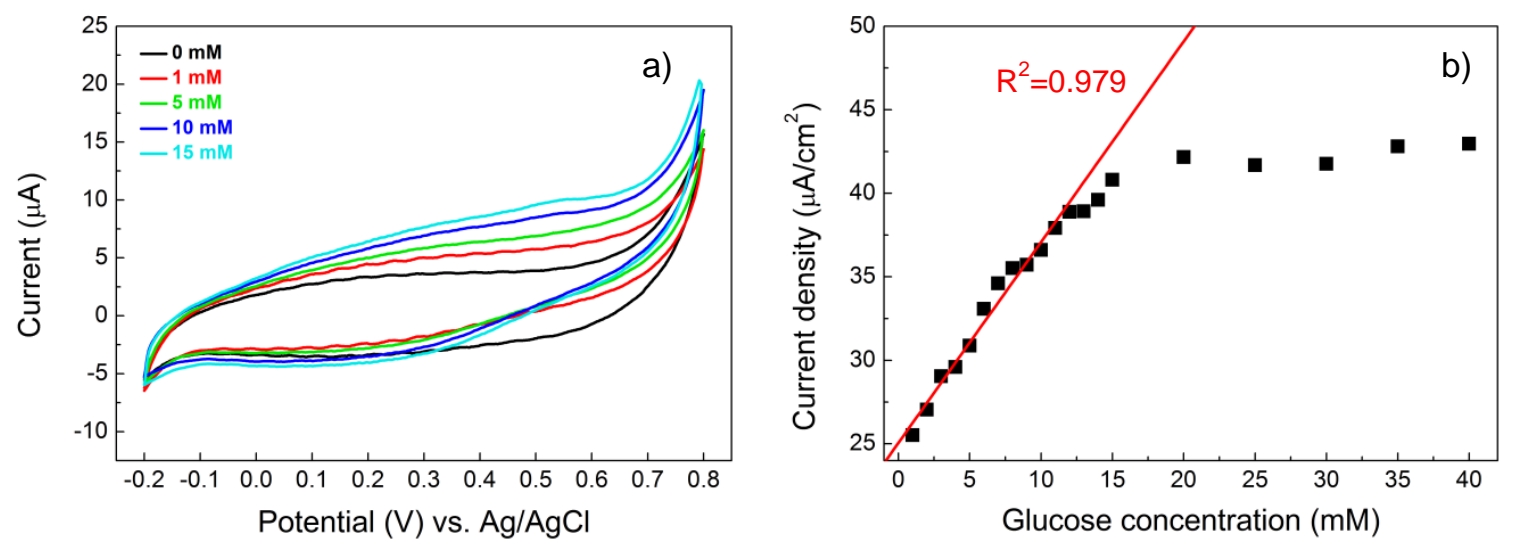

Figure 22. Cyclic voltammogram of a) Au/ZnO-Ts/GOx/Nafion electrode and the current response to various glucose concentrations at the scan rate of $50 \mathrm{mV} / \mathrm{s}$, and d) the deduced calibration curve of current response versus glucose concentration from $1 \mathrm{mM}$ to $40 \mathrm{mM}$ at fixed potential $0.6 \mathrm{~V}$.

It is known that the blood glucose level in the normal range is between 4.4-6.6 $\mathrm{mM} \mathrm{[46],}$ and higher for diabetes patients. It is reported that the current response to glucose concentration saturates at $4.2 \mathrm{mM}$ for nanotubes [29], $4.5 \mathrm{mM}$ for nanocombs [26], and 6.5 $\mathrm{mM}$ for nanotetrapods [33], which barely cover the diagnosis range for diabetes. In this work, the saturation point is 2-3 times higher than those reported in the literature and provides a wider linear monitoring range and a higher limit of detection. The results demonstrate that the $\mathrm{ZnO}$-Ts may serve as a suitable supporting matrix for a wide range of bioreceptors (antibody, enzymes, or nucleic acids) for various biomolecule sensing applications. 


\section{CHAPTER 4}

\section{Conclusions}

$\mathrm{ZnO}$ nano-microtetrapods were synthesized by flame transport synthesis with $\mathrm{Zn}$ precursor microparticles. The size and morphology of the $\mathrm{ZnO}$-Ts were confirmed as four nano-microstructured legs connected together in a tetrahedral symmetry, with each leg exhibiting a tip and stem diameter of $0.7 \mu \mathrm{m}$ and $2.2 \mu \mathrm{m}$ respectively. The PL of ZnO-Ts dispersed in various glucose concentrations and various hydrogen peroxide concentrations was studied. The PL intensity in the UV region decreased with linear dependence on the glucose concentration up to $4 \mathrm{mM}$. PL intensity quenching in the UV region was also observed for hydrogen peroxide with a linear dependence up to roughly $15 \mathrm{mM}$, which indicates hydrogen peroxide as a possible quencher. The attachment of GOx on the $\mathrm{ZnO}$-Ts was confirmed in FT-IR reflectance spectra showing two infrared bands at $1637 \mathrm{~cm}-1$ and $1543 \mathrm{~cm}-1$, which are associated with the peptide bonds in the protein backbone. Cyclic voltammetry showed linear dependence of the current response to the glucose concentrations from $1 \mathrm{mM}$ up to $12 \mathrm{mM}$. The $\mathrm{ZnO}$-Ts demonstrated a wider linear response range and a higher limit of detection than previously reported $\mathrm{ZnO}$ nanotetrapods, which reveal the potential of $\mathrm{ZnO}-\mathrm{Ts}$ for electrochemical glucose sensing. The $\mathrm{ZnO}$-Ts facilitate the platform as supporting matrix to bind other suitable receptors than GOx for various biomolecule sensing applications.

\section{Future works}

In electrochemical glucose biosensors, the attachment of GOx while retaining the bioactivity on a suitable supporting matrix is a crucial factor. GOx can bind with the $\mathrm{ZnO}-$ Ts via physical attachment or chemical binding. The physical attachment of GOx is the electrostatic interactions utilized in this work, which depends on the net surface charge in the reaction medium. The chemical binding of GOx depends on functional groups, such as silanol groups ( $\mathrm{Si}-\mathrm{OH})$. An alternative is to incorporate (3-aminopropyl)trimethoxysilane as cross-linking agent between the GOx and the $\mathrm{ZnO}-\mathrm{Ts}$, as well as the electrode surface.

Another alternative is to incorporate graphene to form $\mathrm{ZnO}$-Ts/Graphene hybrid for the possibility to enhance the electrical, magnetic, and optical properties. 


\section{References}

[1] R. P. Feynman, "There's plenty of room at the bottom," Eng. Sci., vol. 23, no. 5, pp. 22-36, 1960.

[2] T. Hey and P. Walters, The New Quantum Universe, Second edi. Cambridge: Cambridge University Press, 2003.

[3] K. E. Drexler, Engines of Creation: The Coming Era of Nanotechnology. New York: Anchor Books, 1986.

[4] ISO (International Standards Organization), "ISO/TC 229 Nanotechnologies,” 2005. [Online]. Available: http://www.iso.org/iso/iso_technical_committee.html?commid= 381983. [Accessed: 20-May-2015].

[5] G. L. Hornyak, J. Dutta, H. F. Tibbals, and A. K. Rao, Introduction to Nanoscience. Boca Raton: CRC Press, 2008.

[6] M. S. Ramachandra Rao and T. Okada, ZnO Nanocrystals and Allied Materials, vol. 180. New Delhi: Springer India, 2014.

[7] G. Heiland, E. Mollwo, and F. Stöckmann, "Electronic Processes in Zinc Oxide," in Solid State Physics, vol. 8, 1959, pp. 191-323.

[8] R. J. Gettens and G. L. Stout, Painting Materials: A Short Encyclopedia. New York: Dover Publications, Inc., 1966.

[9] C. G. Maier, "Zinc Smelting from a Chemical and Thermodynamic Viewpoint," Washington D.C., 1930.

[10] C. W. Bunn, "The lattice-dimensions of zinc oxide," Proc. Phys. Soc., vol. 47, no. 5, pp. 835-842, 1935.

[11] C. Wagner and W. Schottky, "Theorie der geordneten Mischphasen," Zeitschrift für Phys. Chemie, vol. 11, pp. 163-210, 1930.

[12] C. F. Klingshirn, B. K. Meyer, A. Waag, A. Hoffmann, and J. Geurts, Zinc Oxide, 1st ed., vol. 120. Berlin, Heidelberg: Springer Berlin Heidelberg, 2010.

[13] C. Klingshirn, "ZnO: Material, Physics and Applications," ChemPhysChem, vol. 8, no. 6, pp. 782-803, 2007.

[14] T. Minami, H. Nanto, and S. Takata, "Highly Conductive and Transparent Aluminum Doped Zinc Oxide Thin Films Prepared by RF Magnetron Sputtering," Jpn. J. Appl. Phys., vol. 23, no. Part 2, No. 1, pp. L280-L282, May 1984. 
[15] I. T. Drapak, "Visible luminescence of a $\mathrm{ZnO}-\mathrm{Cu} 2 \mathrm{O}$ heterojunction," Semiconductors, vol. 2, pp. 624-625, 1968.

[16] I. Akasaki, "Blue Light: A Fascinating Journey (Nobel Lecture)," Angew. Chemie Int. Ed., vol. 54, no. 27, pp. 7750-7763, 2015.

[17] H. Amano, M. Kito, K. Hiramatsu, and I. Akasaki, "P-Type Conduction in MgDoped GaN Treated with Low-Energy Electron Beam Irradiation (LEEBI)," Jpn. J. Appl. Phys., vol. 28, no. Part 2, No. 12, pp. L2112-L2114, 1989.

[18] D. C. Look, D. C. Reynolds, J. R. Sizelove, R. L. Jones, C. W. Litton, G. Cantwell, and W. C. Harsch, "Electrical properties of bulk ZnO," Solid State Commun., vol. 105, no. 6, pp. 399-401, 1998.

[19] A. Tsukazaki, A. Ohtomo, and M. Kawasaki, "High-mobility electronic transport in ZnO thin films," Appl. Phys. Lett., vol. 88, no. 15, p. 152106, 2006.

[20] U. Özgür, Y. I. Alivov, C. Liu, a. Teke, M. a. Reshchikov, S. Doğan, V. Avrutin, S.J. Cho, and H. Morkoç, "A comprehensive review of $\mathrm{ZnO}$ materials and devices," J. Appl. Phys., vol. 98, no. 4, p. 041301, 2005.

[21] V. Coleman and C. Jagadish, "Basic Properties and Applications of ZnO," in Zinc Oxide Bulk, Thin Films and Nanostructures, Elsevier, 2006, pp. 1-20.

[22] A. Janotti and C. G. Van de Walle, "Fundamentals of zinc oxide as a semiconductor," Reports Prog. Phys., vol. 72, no. 12, p. 126501, 2009.

[23] C. Hanley, J. Layne, A. Punnoose, K. M. Reddy, I. Coombs, A. Coombs, K. Feris, and D. Wingett, "Preferential killing of cancer cells and activated human T cells using ZnO nanoparticles," Nanotechnology, vol. 19, no. 29, p. $295103,2008$.

[24] K. M. Reddy, K. Feris, J. Bell, D. G. Wingett, C. Hanley, and A. Punnoose, "Selective toxicity of zinc oxide nanoparticles to prokaryotic and eukaryotic systems," Appl. Phys. Lett., vol. 90, no. 21, p. 213902, 2007.

[25] C. Hanley, A. Thurber, C. Hanna, A. Punnoose, J. Zhang, and D. G. Wingett, "The Influences of Cell Type and $\mathrm{ZnO}$ Nanoparticle Size on Immune Cell Cytotoxicity and Cytokine Induction," Nanoscale Res. Lett., vol. 4, no. 12, pp. 1409-1420, 2009.

[26] J. X. Wang, X. W. Sun, a. Wei, Y. Lei, X. P. Cai, C. M. Li, and Z. L. Dong, "Zinc oxide nanocomb biosensor for glucose detection," Appl. Phys. Lett., vol. 88, no. 23, pp. 53-56, 2006. 
[27] X. Liu, Q. Hu, Q. Wu, W. Zhang, Z. Fang, and Q. Xie, “Aligned ZnO nanorods: A useful film to fabricate amperometric glucose biosensor," Colloids Surfaces B Biointerfaces, vol. 74, no. 1, pp. 154-158, 2009.

[28] D. Pradhan, F. Niroui, and K. T. Leung, "High-performance, flexible enzymatic glucose biosensor based on $\mathrm{ZnO}$ nanowires supported on a gold-coated polyester substrate,” ACS Appl. Mater. Interfaces, vol. 2, no. 8, pp. 2409-2412, 2010.

[29] K. Yang, G.-W. She, H. Wang, X.-M. Ou, X.-H. Zhang, C.-S. Lee, and S.-T. Lee, “ZnO Nanotube Arrays as Biosensors for Glucose," J. Phys. Chem. C, vol. 113, no. 47, pp. 20169-20172, 2009.

[30] A. Aboulaich, C.-M. Tilmaciu, C. Merlin, C. Mercier, H. Guilloteau, G. Medjahdi, and R. Schneider, "Physicochemical properties and cellular toxicity of (poly)aminoalkoxysilanes-functionalized $\mathrm{ZnO}$ quantum dots," Nanotechnology, vol. 23, no. 33, p. 335101, 2012.

[31] Y. Zhang, M. K. Ram, E. K. Stefanakos, and D. Y. Goswami, "Synthesis, characterization, and applications of ZnO nanowires," J. Nanomater., vol. 2012, 2012.

[32] A. Kołodziejczak-Radzimska and T. Jesionowski, "Zinc Oxide-From Synthesis to Application: A Review," Materials (Basel)., vol. 7, no. 4, pp. 2833-2881, 2014.

[33] Y. Lei, X. Yan, N. Luo, Y. Song, and Y. Zhang, “ZnO nanotetrapod network as the adsorption layer for the improvement of glucose detection via multiterminal electron-exchange," Colloids Surfaces A Physicochem. Eng. Asp., vol. 361, no. 1-3, pp. 169-173, 2010.

[34] G.-W. She, X.-H. Zhang, W.-S. Shi, X. Fan, J. C. Chang, C.-S. Lee, S.-T. Lee, and C.-H. Liu, "Controlled synthesis of oriented single-crystal $\mathrm{ZnO}$ nanotube arrays on transparent conductive substrates," Appl. Phys. Lett., vol. 92, no. 5, p. 053111, 2008.

[35] L. E. Greene, M. Law, D. H. Tan, M. Montano, J. Goldberger, G. Somorjai, and P. Yang, "General Route to Vertical ZnO Nanowire Arrays Using Textured ZnO Seeds," Nano Lett., vol. 5, no. 7, pp. 1231-1236, 2005.

[36] Q. Wan, T. H. Wang, and J. C. Zhao, "Enhanced photocatalytic activity of ZnO nanotetrapods," Appl. Phys. Lett., vol. 87, no. 2005, pp. 2012-2015, 2005. 
[37] R. Adelung, S. Kaps, Y. K. Mishra, M. Claus, T. Preusse, and C. Wolpert, "Elastic material with a pore space bridged at the particle level by nanobridges between particles," PCT/DE2011/0002822011.

[38] D. Gedamu, I. Paulowicz, S. Kaps, O. Lupan, S. Wille, G. Haidarschin, Y. K. Mishra, and R. Adelung, "Rapid fabrication technique for interpenetrated $\mathrm{ZnO}$ nanotetrapod networks for fast UV sensors," Adv. Mater., vol. 26, no. 10, pp. 1541-1550, 2014.

[39] Q. Zhang, C. S. Dandeneau, X. Zhou, and G. Cao, "ZnO Nanostructures for DyeSensitized Solar Cells," Adv. Mater., vol. 21, no. 41, pp. 4087-4108, 2009.

[40] H. Ohta, M. Hirano, K. Nakahara, H. Maruta, T. Tanabe, M. Kamiya, T. Kamiya, and H. Hosono, "Fabrication and photoresponse of a pn-heterojunction diode composed of transparent oxide semiconductors, p-NiO and n-ZnO,” Appl. Phys. Lett., vol. 83, no. 5, p. 1029, 2003.

[41] M. H. Huang, S. Mao, H. Feick, H. Yan, Y. Wu, H. Kind, E. Weber, R. Russo, and P. Yang, "Room-temperature ultraviolet nanowire nanolasers.," Science, vol. 292, no. 5523, pp. 1897-9, 2001.

[42] S. Sakthivel, B. Neppolian, M. V. Shankar, B. Arabindoo, M. Palanichamy, and V. Murugesan, "Solar photocatalytic degradation of azo dye: comparison of photocatalytic efficiency of $\mathrm{ZnO}$ and TiO2," Sol. Energy Mater. Sol. Cells, vol. 77, no. 1 , pp. $65-82,2003$.

[43] S. N. Sarangi, S. Nozaki, and S. N. Sahu, "ZnO Nanorod-Based Non-Enzymatic Optical Glucose Biosensor,” J. Biomed. Nanotechnol., vol. 11, no. 6, pp. 988-996, 2015 .

[44] Z. L. Wang, "Towards Self-Powered Nanosystems: From Nanogenerators to Nanopiezotronics,” Adv. Funct. Mater., vol. 18, no. 22, pp. 3553-3567, 2008.

[45] S. Wild, G. Roglic, A. Green, R. Sicree, and H. King, "Global Prevalence of Diabetes: Estimates for the year 2000 and projections for 2030," Diabetes Care, vol. 27, no. 5, pp. 1047-1053, 2004.

[46] J. Wang, "Electrochemical glucose biosensors," in Electrochemical Sensors, Biosensors and their Biomedical Applications, Elsevier, 2008, pp. 57-69.

[47] L. C. Clark and C. Lyons, "Electrode systems for continuous monitoring in cardiovascular surgery," Ann. N. Y. Acad. Sci., vol. 102, no. 1, pp. 29-45, 2006. 
[48] S. K. Arya, S. Saha, J. E. Ramirez-Vick, V. Gupta, S. Bhansali, and S. P. Singh, "Recent advances in $\mathrm{ZnO}$ nanostructures and thin films for biosensor applications: Review," Anal. Chim. Acta, vol. 737, pp. 1-21, 2012.

[49] K.-E. Kim, T. G. Kim, and Y.-M. Sung, "Enzyme-conjugated ZnO nanocrystals for collisional quenching-based glucose sensing," CrystEngComm, vol. 14, no. 8, p. 2859, 2012. 

TRITA -ICT-EX-2015:233 